\title{
tic\&société
}

Vol. 10, N 2-3 | 2ème semestre 2016 - 1er semestre 2017

Spécial varia

Quand des immigrés prennent la parole. Appropriation des plateformes de blogging à des fins politiques par des immigrés marocains

\section{Asmaa AZIZI}

\section{(2) OpenEdition}

Journals

Édition électronique

URL : http://journals.openedition.org/ticetsociete/2168

DOI : 10.4000/ticetsociete. 2168

Éditeur

Association ARTIC

Édition imprimée

Pagination : 230-268

Référence électronique

Asmaa AZIZI, «Quand des immigrés prennent la parole. Appropriation des plateformes de blogging à des fins politiques par des immigrés marocains », tic\&société [En ligne], Vol. 10, № 2-3 | 2ème semestre 2016 - 1er semestre 2017, mis en ligne le 30 avril 2017, consulté le 19 avril 2019. URL : http://journals.openedition.org/ticetsociete/2168 ; DOI : 10.4000/ticetsociete.2168 


\title{
Quand des immigrés prennent la parole. Appropriation des plateformes de blogging à des fins politiques par des immigrés marocains
}

\begin{abstract}
Asmaa AZIZI
Asmaa Azizi est docteure en sciences de l'information et de la communication. Elle est chercheure au Groupe de Recherches Interdisciplinaires sur les processus d'Information et de communication (GRIPIC) du Celsa - Paris Sorbonne. Ses recherches portent sur les médias («traditionnels" et «nouveaux») des immigrés, les médias maghrébins (marocains et tunisiens en particulier) et sur les formes d'engagement politique des jeunes et des diasporas. Elle s'est intéressée, dans ses derniers travaux publiés, à la participation politique médiatisée des immigrés marocains en France. Dans ce cadre, elle a mobilisé à la fois des approches diachronique et synchronique et a étudié des corpus de presse papier et des corpus en ligne (blogues, vidéos, sites communautaires). Elle est aussi chargée d'enseignement dans différentes institutions en France et au Maroc, telles que le Celsa et I'ISIC (Institut Supérieur d'Information et de Communication).
\end{abstract}

aziziasmaa@gmail.com 


\title{
Quand des immigrés prennent la parole. Appropriation des plateformes de blogging à des fins politiques par des immigrés marocains
}

Résumé: L'objectif de cet article est d'étudier quelques stratégies mises en place par certains immigrés marocains afin de faire parvenir leurs voix auprès de leurs pairs et des responsables politiques marocains. L'article porte plus précisément sur les appropriations des plateformes de bl gging à des fins politiques. La méthodologie mise en place dans ce travail mobilise principalement l'analyse de discours, l'entretien compréhensif et l'étude de l'énonciation dans ses deux versants éditorial et linguistique. Tout en prenant en compte les contextes politico-médiatiques dans lesquels ces pratiques prennent forme, l'article étudie les phénomènes qui président à la construction du sens politique de ces blogues (stratégies narratives, argumentation, thématiques, etc.) ainsi que le profil des instigateurs de ces espaces. Les pratiques politiques qui s'y donnent à voir s'apparentent à des pratiques médiatiques, discursives et créatives. Elles sont caractérisées par la plasticité de leurs formes et par leurs caractères dénonciateurs et critiques. Elles prennent la forme d'un refus et promettent de donner une existence et une visibilité à ces immigrés.

Mots clés: immigrés, Maroc, blogue, pratique politique en ligne, prise de parole.

\begin{abstract}
This paper analyzes several strategies of some Moroccan immigrants who aim to speak to their peers and to Moroccan politicians. It focuses specifically on the political appropriation of blogging spaces. The methodology developed in this work mainly mobilizes discourse analysis, comprehensive interviews and the study of enunciation (editorial and linguistic). This work takes into account the political and media environments in which these practices take shape. It examines the different phenomena that govern the construction of political meaning in these blogs by studying on one hand narrative strategies, argumentation and topics and, on the other hand, the profile of the instigators of these spaces. These political practices are media related, discursive and creative. They are characterized by the diversity of their forms and by their critical tone of denunciation. They promise to give an existence and visibility to these immigrants.
\end{abstract}

Keywords: immigrants, Morocco, blog, online political practices, speaking. 
Quand des immigrés prennent la par'le. Appropriation des plateformes de $b l$ 'gging à des fins politiques par des immigrés marocains

Resumen: El objetivo del presente artículo es estudiar algunas de las estrategias desarrolladas por ciertos emigrantes marroquíes que tienen por finalidad hacerse escuchar por sus pares y por los responsables políticos de su país. En concreto, el texto se interesa por las apropiaciones de las plataformas de blogging con fines políticos. La metodología aplicada en este trabajo moviliza principalmente el análisis del discurso, la "entrevista comprensiva" y el estudio de la enunciación en sus dos vertientes, -editorial y lingüística-. Tomando en cuenta los contextos político-mediáticos en los cuales estas prácticas toman forma, el artículo estudia los fenómenos que presiden la construcción del sentido político de estos blogs (estrategias narrativas, argumentación, temáticas, etc.), así como el perfil de los responsables de tales espacios. Las prácticas políticas que se muestran tienen un parentesco con las prácticas mediáticas, discursivas y creativas. Dichas prácticas se caracterizan por la plasticidad de sus formas y por su tendencia denunciante y crítica. Planteándose como una resistencia, ellas prometen conferir una existencia y una visibilidad a esos inmigrantes.

Palabras claves : emigrantes, Marruecos, blog, práctica política en la web, tomar la palabra. 
Depuis la deuxième moitié des années 1990, le nombre de travaux de recherche étudiant les relations qu'entretiennent les immigrés avec les technologies de l'information et de la communication (TIC) n'a cessé de croître. La revue tic\&s ciété a consacré, en 2009, un double numéro à ce sujet. Coordonné par Tristan Mattelart ${ }^{1}$, ce dossier a le mérite, d'un côté, de proposer une lecture critique d'une partie de la littérature de ce champ de recherche et de dégager, de l'autre, la diversité des angles à partir desquels la relation entre TIC et immigration/émigration a pu être appréhendée.

Dans la continuité de ces travaux, nous nous proposons d'étudier les appropriations des médias informatisés ${ }^{2}$ par les immigrés à des fins politiques. Notre questionnement de départ est déterminé par les spécificités du terrain de cette recherche, à savoir le contexte marocain : c'mment des immigrés mar' cains, depuis leur pays d'installati $n$, s'eff' rcent-ils de faire entendre leur $v^{\prime} i x^{3}$ au Mar' $c$ ? Telle est la question que nous nous posons et qui nous guidera tout au long de ce travail. L'objectif de ce texte est de cerner et de comprendre ce que nous désignons par "les pratiques politiques en ligne " de ces immigrés. Aussi, il vise à appréhender les différentes stratégies d'appropriation des médias informatisés mises en place, et ce, pour s'informer, commenter l'actualité et dénoncer les dysfonctionnements de la politique sociale et économique de leur pays d'origine.

Avant d'aborder le sens de l'expression de «pratiques politiques en ligne » des immigrés marocains que nous mobilisons dans le cadre de ce travail, un bref rappel de l'histoire de la participation politique des immigrés marocains

\footnotetext{
${ }^{1} \mathrm{~N}^{\circ} 1-2$, vol. 3, 2009.

${ }^{2}$ Notion utilisée ici pour remplacer l'expression « technologies de l'information et de la communication » qui « occulte tout un versant de la fonction sociale et du fonctionnement des médias » (Davallon, 1992, p. 102). La notion de " médias informatisés » fait référence à des " dispositifs techniques " ayant pour constituants des appareils de traitement de l'information, au sens mathématique du terme, et ayant pour effet social de faire circuler des messages et, par là, de rendre possible des échanges d'information, des interprétations, des productions de connaissances et de savoirs dans la société (Jeanneret, 2007). Ils sont avant tout des médias, c'està-dire des « dispositifs permettant les échanges signifiants entre les hommes et conditionnant d'une certaine façon la forme de ces échanges » (Jeanneret, 2007, p. 92).

${ }^{3}$ Dans cet article, avec l'usage des deux termes $v^{\prime}$ ix et par’ $l e$, nous puisons dans la métaphore de l'oralité, sans toutefois faire le deuil du dispositif technique des espaces où circule cette " parole ». Olivier Aim et Yves Jeanneret (2007) affirment que, " même si le commentaire est obsédé par l'oralité (prise de parole, scène, dialogue, etc.), le dispositif configure un tissu dense d'écriture » (p. 70). Dans le même sens, Samira Ouardi (2006) souligne que « la prise de parole est une prise d'écriture » (p. 63).
} 
Quand des immigrés prennent la par'le. Appropriation des plateformes de $b /$ ' gging à des fins politiques par des immigrés marocains

semble nécessaire. Ce rappel permettra également de saisir la symbolique et le sens de ces pratiques politiques en ligne.

Dans son article intitulé "L'engagement citoyen des Marocains de l'étranger ", Thomas Lacroix (2005) dresse un tableau des pratiques politiques transnationales ${ }^{4}$ des immigrés marocains depuis les années 1960. II précise que l'entrée dans le champ politique du pays d'origine a longtemps été contrôlée par le régime marocain à l'échelle nationale et par les structures notabiliaires à l'échelon local. En se livrant au même exercice, Antoine Dumont (2008) affirme que les immigrés marocains sont " $v$ ` iceless ", sans voix, étant donné l'exclusion du champ politique dont ils font l'objet à la fois dans leurs pays d'origine et d'installation (Dumont, 2008).

En effet, les multiples tensions et conflits idéologiques entre la monarchie d'un côté et les exilés politiques ${ }^{5}$, de l'autre, ont conduit au verrouillage du champ politique marocain par la première, qui a exclu ses émigrés de toutes formes de participation politique. Et Lacroix (2005) d'affirmer que

"l'évolution des pratiques citoyennes des Marocains de l'étranger a été marquée par l'expérience des engagements associatifs et syndicaux au sein de la société d'accueil mais également par un changement des échelles d'action dans la société d'origine; du politique vers l'infra-politique, du national vers le translocal » (p. 89).

Autrement dit, ces immigrés ont mis en place une forme de citoyenneté novatrice prenant en compte leur double appartenance : tout en revendiquant le droit de vote au sein des sociétés d'installation, ils mettent en place un ensemble de pratiques relevant, comme le désigne Lacroix, du registre de l'infra-politique (soutien scolaire, permanence sociale au sein des associations d'immigrés, lutte pour les sans-papiers, etc.).

$\mathrm{Si}$ «la politique d'hostilité » (Belguendouz, 2006) qui a longtemps caractérisé les relations entre l'État marocain et ses immigrés a cédé la place à "une politique de réconciliation ${ }^{6}$ " (ibid.) à partir des années 1990, il n'en demeure pas moins que

\footnotetext{
${ }^{4}$ Par ces dernières, il faudrait entendre les activités mises en place par les immigrés pour participer aux politiques nationales ou étrangères de leur pays d'origine.

${ }^{5}$ Notamment lors de la fin des années 1960 et au cours des années 1970 et 1980.

${ }^{6}$ Politique ayant vu le jour dans les années 1990 sous le règne de Hassan II. Elle est caractérisée par la mise en place de nombreuses institutions en charge de la question migratoire (telles que la Fondation Mohamed $\mathrm{V}$ pour les Marocains résidant à l'étranger (MRE), le Ministère chargé de la communauté marocaine à l'étranger, la Banque Al Amal et, plus récemment, le Conseil de la communauté marocaine à l'étranger (CCME)).
} 
la participation politique des Marocains de l'étranger reste le parent pauvre de cette réconciliation ${ }^{7}$.

Étant donné ces éléments de contexte et par conséquent les marges de manœuvre dont disposent les immigrés marocains, nous pensons que leurs actions politiques ne peuvent être appréhendées uniquement sous l'angle des formes classiques d'engagement. Le passage à l'action politique signifie pour beaucoup d'immigrés la prise de parole (Memmi, 1985). Memmi (1985) précise qu'en « dehors des décisions politiques émanant d'un très petit nombre de professionnels de la politique ayant un pouvoir d'initiative, en quoi consiste l'activité politique ? En une production et reproduction de discours » (p. 310). Nous avançons qu'il existe d'autres tactiques significatives pour faire de la politique pour des acteurs qui agissent et négocient une place dans le champ politique duquel ils sont exclus. Nous désignons ici ces manières autres de faire de la politique par "pratiques politiques en ligne". Ces pratiques sont essentiellement discursives. Elles naissent d'une production et d'une reproduction de discours. Elles sont médiatiques, car elles prennent forme et sens dans et entre des espaces médiatiques différents, en l'occurrence des espaces en ligne. Elles sont créatives: la créativité doit être perçue comme intimement liée au geste d'appropriation regroupant des actions de dé-sémiotisation et de re-sémiotisation des médias informatisés, ici, à des fins de participation politique. Autrement dit, il s'agit de toutes les pratiques d'écriture, de réécriture, de transformation et de détournement d'un dispositif communicationnel par divers acteurs sociaux à des fins politiques $^{8}$.

Ces pratiques s'apparentent à celles s'inscrivant dans le registre de la " politique informelle » (LeGall, Offerlé et Ploux, 2012). Elles sont non institutionnelles et mises en place par la marge, en l'occurrence les immigrés. Si ces pratiques peuvent paraître illégitimes ou triviales pour un lecteur occidental, elles disposent toutefois d'une dimension symbolique importante compte tenu de leur contexte de production et des marges de manœuvre dont disposent leurs instigateurs.

\footnotetext{
${ }^{7}$ Ainsi, les immigrés marocains n'ont pas pu participer à la dernière élection législative en date, à savoir celle du 7 octobre 2016.

${ }^{8}$ Dans ce sens, Victoria Bernal (2006) précise que les espaces web, où circule une parole politique, sont à la fois consommés et créés par les internautes. Elle invite à les penser en tant que productions culturelles des immigrés. Selon elle, toute une dimension significative de ces pratiques serait perdue " if they [are] underst ' $d$ simply in the instrumental $p$ ' litical terms ' $f$ rallying supp ' $r t^{\prime} r$ expressing dissent » (p. 170). Traduction libre de « si elles sont considérées simplement dans leur dimension instrumentale de rassembler du soutien ou d'exprimer la dissidence »).
} 
Quand des immigrés prennent la par'le. Appropriation des plateformes de $b /$ ' gging à des fins politiques par des immigrés marocains

Par ailleurs, le désenchantement des Marocains, au Maroc et à l'extérieur, vis-à-vis des médias traditionnels est grand ${ }^{9}$. Dans un contexte où les médias marocains sont sous la mainmise du pouvoir ${ }^{10}$ et où l'émergence d'espaces médiatiques "indépendants " se voit réduite, le web, à travers ses promesses ${ }^{11}$, apparaît pour les Marocains au Maroc et à l'étranger comme un espace de premier choix de libre expression, de démocratisation et de concrétisation des idéaux politiques. Un autre désenchantement touchant cette fois-ci le champ politique est à noter. En sont la preuve les résultats des élections législatives au Maroc. Ainsi, le taux d'absentéisme lors des élections législatives de 2007 a atteint le taux record de $63 \%$. Quant aux taux de participation aux élections d'octobre 2011 et 2016, il a été respectivement de $45 \%$ et $43 \%$.

Quelles motivations et significations symboliques président à ces pratiques politiques en ligne des immigrés ? Quels sont les phénomènes qui participent à la construction du sens politique de ces blogues? Quelles sont les thématiques traitées dans ces espaces en ligne? Quelles formes prennent ces discours? Qui consulte ces espaces ? Quel est le profil des immigrés instigateurs de ces pratiques ? De quelle manière la figure du blogueur-énonciateur se manifeste-t-elle dans les énoncés de ces blogues? Quels positionnements se donnent ces internautes par rapport aux médias mainstream? Telles sont quelques-unes des questions que nous nous posons et qui seront traitées dans ce qui suit.

Nous proposons d'aborder cette question de la participation politique en ligne des immigrés marocains à travers une approche communicationnelle. Cette approche a le mérite d'un côté de rendre compte du rôle actif des immigrés dans l'appropriation des espaces en ligne, en l'occurrence les blogues. De l'autre côté, elle ne considère pas la communication dans sa dimension instrumentale, c'est-à-dire en tant qu' " outil, au mieux pédagogique au pire manipulatoire » (Monnoyer-Smith, 2007, p. 36), comme c'est le

\footnotetext{
${ }^{9}$ La presse marocaine de la période allant de 1960 à 1990 est principalement « une presse partisane bilingue, en plein ess ' $r$ mais muselée » (Daghmi, Pulvar et Toumi, 2012). Quant à la communication audiovisuelle, elle représente depuis l'indépendance une des stratégies de domination du régime politique.

${ }^{10}$ Et où la liberté de la presse est de plus en plus réduite. A ce propos, selon Reporters sans frontières, le Maroc est classé en 2016 à la $131^{\mathrm{eme}}$ position sur un total de 180 pays.

${ }^{11}$ A savoir l'imaginaire de l'horizontalité (qui permettrait le passage d'un modèle de communication " un vers tous » à un modèle de communication "tous vers tous "), de l'accessibilité (qui permettrait un accès à moindre coût et de n'importe quel endroit), de l'égalité (qui permettrait à n'importe qui d'y accéder) et de l'ouverture (qui ferait principalement référence à l'idéal de transparence) (Flichy, 2001).
} 
cas de nombreux travaux en sciences politiques. II est question, ici, de projeter un regard communicationnel sur un objet politique sans pour autant « instrumentaliser la communication ou désubstantialiser le politique » (ibid., p. 24).

Puisqu'on a affaire essentiellement à des discours qui circulent dans ces espaces en ligne, nous mobiliserons une analyse de l'énonciation dans ses deux versants linguistique et éditorial. Le versant éditorial repose sur les travaux d'Emmanuel Souchier (1998) ${ }^{12}$, qui octroie un intérêt particulier à l'agencement formel et éditorial du texte, ici, des blogues. Cette énonciation permettra de comprendre comment le dispositif informe le sens des discours qui y circulent. Quant à l'énonciation dans son versant linguistique, elle s'appuie principalement sur l'analyse de discours circulant dans trois blogues retenus dans notre corpus. Elle permettra d'analyser une pluralité de stratégies mises en place par les instances énonciatrices de ces blogues. Afin d'éviter les écueils de l'analyse de discours comme méthodologie, nous avons eu recours à une série d'entretiens compréhensifs. Nous avons réalisé sept entretiens avec des blogueurs immigrés marocains $^{13}$.

Le corpus de l'étude ne se veut ni représentatif ni exhaustif de l'ensemble des pratiques politiques en ligne de ces immigrés. Pour autant et malgré son caractère lacunaire, il en est significatif. Nous nous limiterons en l'occurrence à l'étude des pratiques se déroulant au sein d'espaces de bl'gging. Ceux-ci sont parmi les premiers espaces web à être appropriés et visités, à la fois par les immigrés et les Marocains au sol. Ces " machines textuelles ${ }^{14}$ " (Souchier, Jeanneret, Le Marec, 2003 , p. 25) se déclinent en différentes catégories et projets éditoriaux ${ }^{15}$. Notre corpus s'est aussi vu alimenter par un

\footnotetext{
${ }^{12}$ De nombreux travaux de recherche s'intéressant à la question des liens entre web et politique font le deuil de l'étude des dispositifs technosémiotiques. L'usage de plus en plus fréquent de la métaphore de l'oralité avec des expressions telles que " prise de parole », " voix », etc., rend la question du dispositif transparente.

${ }^{13}$ Nous avons établi une liste de blogues d'immigrés marocains. La constitution de cette liste s'est basée sur les résultats du concours $M^{\prime} r^{\prime} c c^{\prime} B l^{\prime} g s$ Awards, lancé en 2007, sur la visibilité médiatique dont bénéficiaient certains blogues d'immigrés ainsi que sur les liens vers des blogues figurant dans la $b l^{\prime} g r^{\prime}$ II (liste de blogues favoris). Nous avons contacté par mails les blogueurs-éditeurs de la liste et sept d'entre eux ont accepté de nous accorder un entretien en face à face.

${ }^{14}$ Ces machines textuelles font référence aux machines « auxquelles on accède et que l'on manipule à travers et par l'écriture » (Souchier, Jeanneret, Le Marec, 2003, p. 25).

${ }_{15}$ À titre d'exemple et en dehors de la catégorie « blogues politiques » qui nous intéresse ici, on trouve également des blogues littéraires, des blogues culinaires, des blogues d'humeur, etc. L'appropriation importante de ce dispositif par les Marocains au Maroc et les immigrés fût le déclencheur de l'organisation d'un concours annuel, le $M^{\prime} r^{\prime} c c^{\prime} B l^{\prime} g s$ Awards. Dans une tentative d'adaptation aux nouvelles
} 
Quand des immigrés prennent la par 'le. Appropriation des plateformes de $b /$ ' gging à des fins politiques par des immigrés marocains

ensemble de billets issus d'autres blogues d'immigrés, d'articles de presse ainsi que par quelques pages disponibles sur le site web dit de réseau social Facebook et le site de micro-bl' gging Twitter.

Notre argumentation au sein de cet article s'articule autour de deux principaux points faisant l'objet chacun d'une partie. La première partie propose une genèse des pratiques politiques en ligne des immigrés marocains. Elle vise à donner une épaisseur historique aux pratiques de b/ gging étudiées dans la seconde partie. Celle-ci étudie, d'un côté, les différentes stratégies narratives et argumentaires ainsi que les caractéristiques de ces espaces de bl'gging. Elle dresse, d'un autre côté, une esquisse du profil des instigateurs de ces blogues politiques.

\section{Genèse des pratiques politiques en ligne des immigrés marocains}

Si l'objectif de cette partie est d'esquisser une genèse des pratiques politiques en ligne des immigrés marocains, nous estimons toutefois utile de rappeler que celles-ci s'inscrivent dans la continuité de pratiques se déroulant " hors ligne " et qui les précédaient. Tristan Mattelart (2007) a souligné que " les moyens de communication sont depuis longtemps utilisés par l'émigration pour essayer d'influencer à distance la vie politique du pays quitté » (p. 25). En effet, les immigrés marocains n'ont pas attendu l'avènement d'Internet pour participer, à distance, à la vie politique de leur pays d'origine. Dès les années 1930, plusieurs titres de presse, faits d'acteurs hétérogènes (étudiants, exilés politiques et ouvriers), disposant de différents formats éditoriaux et de périodicité (journaux, revues, bulletins d'associations, etc.) et puisant dans une diversité de modèles économiques (dons, financement de particuliers et d'hommes politiques, abonnement, etc.), ont vu le jour (Azizi, 2016). Ces titres de presse représentaient des espaces de circulation d'un discours politique portant sur différentes thématiques relatives au pays d'origine (despotisme politique, exil et immigration, éducation et santé, colonialisme, etc.). Aussi, ces espaces donnaient à voir les expressions d'appartenance de ces immigrés et leurs différentes stratégies de représentation d'un soi collectif.

II n'est pas de notre propos d'établir un inventaire chronologique précis et exhaustif des différentes pratiques politiques en ligne des immigrés marocains. En effet, la difficulté de repérage de l'intégralité de ces pratiques est

plateformes web, ce concours, lancé en 2007, prime des vidéos YouTube, des comptes Twitter, des comptes Instagram, etc. 
inhérente aux caractéristiques propres du web, à savoir la labilité et la volatilité de ses contenus. Ainsi, cette esquisse de genèse est proposée uniquement à titre indicatif et se veut comme un cadre contextuel d'inscription des pratiques qui seront abordées ultérieurement. Seules les pratiques ayant eu une grande envergure sont abordées dans cet historique. II faut entendre par là les pratiques ayant pu mobiliser un grand nombre d'acteurs sociaux, ou celles ayant conduit à une grande médiatisation à l'échelle nationale, voire internationale, mais aussi celles ayant pu avoir un impact auprès des autorités politiques marocaines. C'est essentiellement à travers les pratiques exposées ci-dessous qu'un large nombre de Marocains, internautes ou non, ont pris connaissance de l'existence de telles appropriations politiques du web. De surcroît, les exemples retenus donnent à voir la complémentarité entre les pratiques politiques des immigrés et celles des Marocains au Maroc. En effet, nous considérons que les frontières entre les pratiques en ligne des Marocains au Maroc et celles des immigrés sont perméables. Le caractère du web, que d'aucuns désignent de " transnational », permettrait à ces internautes de consulter et de commenter les mêmes espaces. À l'instar de tout dispositif communicationnel, les médias informatisés affectent les coordonnées spatiotemporelles. Ils permettraient ainsi une réduction des distances géographiques en permettant à des internautes situés dans des zones géographiques différentes d'échanger (Latzko-Toth et Proulx, 2013). Ainsi, les exemples ci-dessous présentent des pratiques tantôt initiées par des Marocains au Maroc, tantôt par des immigrés marocains.

C'est à l'année 2007 que remonte la première pratique politique en ligne de grande envergure des Marocains. Elle est liée au tournage d'un ensemble de vidéos-amateurs, dont la première est postée en juillet de la même année par un certain Targuist Sniper ${ }^{16}$. Ce " tireur » de la ville de Targuist n'hésitait pas à filmer en flagrant délit de corruption ses " proies », ici des agents de la gendarmerie royale, pour ensuite publier ses vidéos sur la plateforme de partage en ligne YouTube. Ces vidéos sont composées chacune d'une seule séquence sans aucun montage. Leur mauvaise qualité vaut, pour reprendre Sophie Pène (2007), vérité. À titre d'exemple, la première vidéo de cette série a été visionnée, potentiellement ${ }^{17}, 350000$ fois en une semaine. Lors de leurs circulations en ligne, ces vidéos ont emprunté un circuit médiatique varié. Ainsi, en juillet de

\footnotetext{
${ }^{16}$ Vidéo disponible sur le lien <https://www.youtube.com/watch?v=adWfM0J0_ik>, dernière consultation le 23 mars 2017.

${ }^{17} \mathrm{Ce}$ chiffre correspond plus précisément au nombre de clics sur le bouton de lecture, voire au nombre d'ouvertures de la page si la lecture est automatisée.
} 
Quand des immigrés prennent la par'le. Appropriation des plateformes de $b /$ ' gging à des fins politiques par des immigrés marocains

l'année 2007, plusieurs blogues (d'immigrés et de Marocains au Maroc), de sites d'informations en ligne, de sites web communautaires $^{18}$ (Yabiladi.com ${ }^{19}$, Bladi.net ${ }^{20}$ ) ont commenté ces vidéos en les saluant ou les critiquant et en participant par conséquent à leurs circulations médiatiques et à leurs visibilités. Aussi, une presse marocaine, et précisément celle s'autodésignant de "presse indépendante " ${ }^{21}$, ainsi que quelques titres d'une presse internationale ont consacré de nombreux articles à celui que l'on dénomme désormais le "Zorro du web ${ }^{22}$ " devant le silence des médias officiels marocains ${ }^{23}$.

Outre cette héroïsation de la figure du Sniper qui a choisi l'anonymat ${ }^{24}$ comme stratégie d'action et de dénonciation, tout un discours d'enchantement et de fascination par rapport à cette "nouvelle » forme d'activisme a accompagné la circulation des vidéos. Nous étions encore loin du « printemps arabe ». Pourtant, on parlait déjà à cette date des "pouvoirs émancipateurs " du web ainsi que d'une "révolution YouTube ${ }^{25} »$ en cours.

YouTube regorge d'un grand nombre de productions créatives et similaires dans leur dimension dénonciatrice ${ }^{26}$ à celles du Sniper de Targuist. En effet, de plus en plus d'internautes marocains (au Maroc et à l'étranger), à titre individuel, s'approprient ce média informatisé comme une

\footnotetext{
${ }^{18}$ Ces sites web communautaires ont pour « vocation d'informer la communauté ciblée, de la présenter en lui accordant une place dans la sphère médiatique, de s'intéresser à ses problèmes, de construire l'identité culturelle en gardant le lien social avec le pays d'origine et surtout de favoriser son intégration dans la société d'accueil » (Touhami, 2007, p. 114)

19 « TelQuel:Sur la piste du sniper de Targuist », yabiladi, <http://www.yabiladi.com/forum/telquel-piste-sniper-targuist-2-2120240.html>, dernière consultation le 18 mars 2017.

${ }^{20}$ TELQUEL, (2017, 14 octobre), « Sur la piste du sniper de Targuist », bladi.net, <http://www.bladi.net/sniper-de-targuist.html>, dernière consultation le 18 mars 2017.

${ }^{21}$ Comme, à titre d'exemple, le magazine francophone Telquel. : TELQUEL, (2012, 22 février), « Le retour du sniper », telquel.ma, <http://telquel.ma/2012/02/22/leretour-du-sniper_175>, dernière consultation le 18 mars 2017.

22 <http://www.larbi.org/post/2007/07/17/402-maroc-la-revolution-youtube>, dernière consultation le 30 avril 2014.

${ }^{23}$ La chaîne $2 \mathrm{M}$ a uniquement signalé, lors d'une édition du JT, que des membres de la gendarmerie royale avaient été arrêtés suite à un délit de corruption, sans pour autant donner davantage de détails sur l'affaire.

${ }^{24}$ Le Sniper, comme il s'auto-désignait lui-même, est resté anonyme jusqu'en 2013.

En date de publication de cet article, il est en asile politique en Suisse suite aux différents harcèlements qu'il a subis de la part des autorités.

${ }^{25}<$ www.larbi.org/post/2007/07/17/402-maroc-la-revolution-youtube>, dernière consultation le 30 avril 2014 [le billet a été supprimé par l'auteur depuis].

${ }^{26}$ La dernière en date au moment de la publication de cet article est la vidéo d'un jeune homme dénonçant l'état des routes de son petit village.
} 
plateforme de médiati(sati)on pour dénoncer la corruption et d'autres abus ou dysfonctionnements ${ }^{27}$.

La seconde pratique politique en ligne $e^{28}$ de cette genèse fait, quant à elle, suite à l'arrestation et à l'emprisonnement d'un jeune ingénieur marocain du nom de Fouad Mourtada. Ce dernier a créé en 2008 un faux profil sur Facebook au nom du frère du roi Mohammed VI, le prince Rachid. II a été poursuivi et inculpé pour délit de "manque de respect à la famille royale ». $\mathrm{Ce}$ qui était inédit, outre le délit lui-même ${ }^{29}$, est la forte mobilisation qui a fait suite à l'arrestation de Fouad. En effet, et en s'inspirant des répertoires d'actions classiques (Tilly, 1984), une campagne de soutien en ligne a été organisée à la suite d'appels d'internautes marocains, notamment de blogueurs et de quelques militants des droits de l'Homme. A nouveau, une variété de médias informatisés a été mobilisée pour faire circuler l'information concernant cet emprisonnement. Ainsi, des blogues de Marocains (sédentaires et immigrés) et ceux d'acteurs internationaux de la société civile et du monde associatif ont joué un rôle crucial dans la médiatisation de cette arrestation. Outre leur rôle de relais d'information, ces blogues ont servi d'agrégateurs d'information. On y publiait en effet, et en différentes langues, le déroulement et l'avancée du procès, et ce, face au black- "ut médiatique total des télévisions et de la presse officielle. De plus, un groupe de soutien à Fouad Mourtada a été créé sur Facebook et relayait lui aussi les informations et les photos liées à cette campagne de soutien. En plus de ces mobilisations sur le web, coordonnées essentiellement par des immigrés marocains, des manifestations ont été organisées dans plusieurs villes à l'échelle mondiale ${ }^{30}$. L'importante et efficace médiatisation de cette arrestation a fait entrer en jeu les organisations internationales de défense des droits de l'Homme (dont Amnistie internationale $\left.{ }^{31}\right)$, mais aussi une couverture

\footnotetext{
${ }^{27}$ Une sorte de tribune pour s'adresser directement au roi Mohamed VI et lui faire parvenir les doléances de tout un chacun. Ce geste montre le désenchantement d'un certain nombre de Marocains de la justice du pays et octroie en même temps au roi cette qualité de « justice».

${ }^{28}$ Cet exemple illustre le passage d'une pratique individuelle dénuée a pri ri de tout trait politique à une pratique politique et collective.

${ }^{29}$ On parle ainsi dans certaines tribunes du cas du « premier prisonnier Facebook au monde ».

${ }^{30}$ À titre d'exemple, quelques photos des manifestations ayant eu lieu à Paris sont disponibles sur Flickr:

<https://www.flickr.com/photos/24156360@N07/2303484248/?rb=1>, dernière consultation le 18 mars 2017.

${ }^{31}$ AMNESTY INTERNATIONAL, (2008, 27 février), « Trois ans de prison pour avoir créé le profil d'un prince marocain sur Facebook », Amnesty internati nal, $<$ https://www.amnesty.org/fr/latest/news/2008/02/three-years-profiling-moroccanprince-facebook-20080227/>, dernière consultation le 18 mars 2017.
} 
Quand des immigrés prennent la par 'le. Appropriation des plateformes de $b /$ ' gging à des fins politiques par des immigrés marocains

médiatique assurée par des titres de presse internationale. En parallèle, une campagne de "blogues en grève ${ }^{32}$ " s'inspirant elle aussi directement des répertoires d'action hors ligne a été lancée. Finalement, Fouad Mourtada a été relaxé après 43 jours d'emprisonnement par " grâce royale ".

Le même scénario de mobilisation est adopté lors de la campagne internationale de soutien au blogueur Erraji, coordonnée elle aussi, entre autres, par des immigrés marocains. En application du code de la presse, ce dernier est condamné à deux ans de prison le 8 septembre 2008 à la suite de sa publication en ligne d'un article critique de la politique sociale de Mohammed VI. Encore une fois, une multiplicité d'acteurs (blogueurs marocains immigrés et sédentaires, ONG de défense des droits de l'Homme, presse internationale et nationale), ainsi qu'une diversité de médias informatisés mobilisés (sites web, blogues, pages Facebook, vidéos YouTube, etc., dédiés à l'affaire "helperraji.com ») caractérisent cette campagne de soutien au blogueur et, plus généralement, de dénonciation de la situation de la liberté d'expression au Maroc. L'usage des photos (Figures 1 à 3) comme stratégie de revendication de la liberté du blogueur emprisonné était très utilisé. Cette pratique, qui peut être qualifiée de création mimétique, consistait à se prendre en photo à côté de la mention "Liberté à Erraji ». Ainsi, des photos circulaient sur le web, où on pouvait identifier des internautes, leur nom ou leur prénom, leur ville d'installation ainsi que la revendication, en différentes langues, de la libération d'Erraji. Ces photos laissent voir que ces mobilisations étaient le fait de Marocains au Maroc, de migrants marocains installés dans différents pays (France, Pologne, Allemagne, États-Unis, Chili, etc.) et de sympathisants ou d'activistes internationaux. Finalement, le blogueur Erraji a finalement été relaxé le 18 septembre 2008.

\footnotetext{
${ }^{32}$ On lit par exemple, dans le blogue d'Abmoul, immigré marocain au Canada : « Demain, Mardi 19 février, ce blog (comme beaucoup d'autres j'espère) sera en grève [...] en protestation contre le procès intenté à Fouad Mourtada et pour appeler à sa libération immédiate et inconditionnelle »

(<http://sonofwords.blogspot.com/2008/02/rage-et-mpris.html?view=classic>, dernière consultation le 18 mars 2017). De même, on trouve dans ce blogue une invitation adressée à l'intention des blogueurs immigrés, leur proposant de solliciter des ONG et d'autres associations des droits de l'Homme de leurs pays d'installation, et ce, pour soutenir des syndicalistes et des militants des droits de l'Homme emprisonnés au Maroc le $1^{\mathrm{er}}$ mai 2007. Le blogueur écrit ainsi : « J'invite aussi (chose que je ferai de ma part) nos blogueurs à l'étranger de contacter les associations locales et internationales des droits de la personne » (http://sonofwords.blogspot.com/2007/06/shame-on-you-let-them-free.html, dernière consultation le 18 mars 2017).
} 


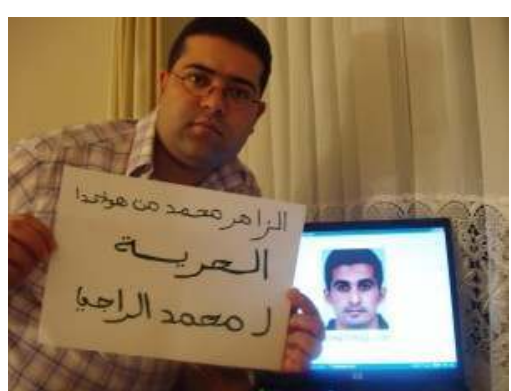

Figure 2. Soutien d'un immigré Marocain installé aux Pays-Bas

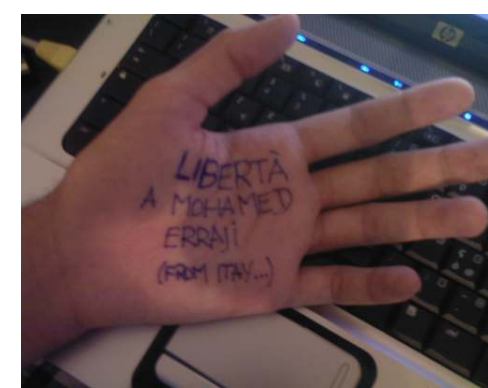

Figure 1 Soutien d'un immigré Marocain installé en Italie

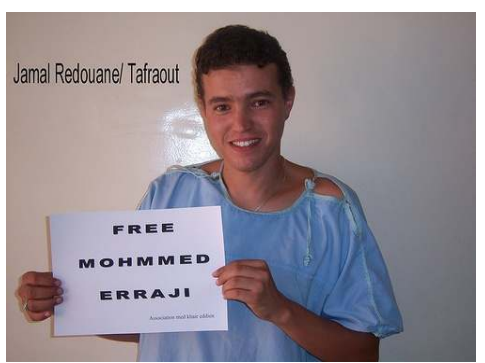

Figure 3. Soutien d'un Marocain au Maroc, de la ville de Tafraout

Le dernier exemple proposé dans le présent historique fait suite à la censure imposée par le gouvernement marocain le 4 août 2009 à deux magazines marocains Telquel et Nichane (version arabophone de Telquel) et du journal français Le $M$ 'nde. Cette censure fait suite à la publication par ces titres des résultats d'un sondage qu'ils ont commandité et où les Marocains étaient invités à évaluer les dix premières années du règne de Mohammed VI. En dépit des résultats de ce sondage favorable à la monarchie, la censure est tombée. En effet, ces résultats montraient que $91 \%$ des Marocains approuvaient la politique menée par Mohammed VI. Ainsi, une campagne en ligne dénonçant cette censure est lancée sous le slogan « Je suis un $9 \%$ » (Figure 4 ). 
Quand des immigrés prennent la par'le. Appropriation des plateformes de $b /$ ' gging à des fins politiques par des immigrés marocains

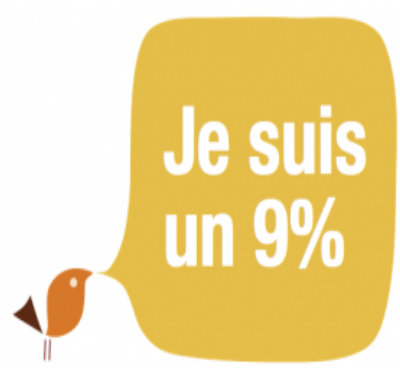

Figure 4. Logo du

« mouvement » « Je suis un $9 \% »$

Cet acte d'énonciation a une valeur performative explicite : par l'acte même de sa profération, de son écriture, de son apposition sur sa photo de profil personnel (de blogue, de page Facebook), l'internaute revendique sa désapprobation de la politique royale officielle. L'initiateur de cette campagne est Anas Alaoui, un blogueur marocain installé en France qui déclare, dans un entretien que nous avons pu mener avec lui, son étonnement quant à la large diffusion du slogan et de l'avatar « Je suis un $9 \%$ ». II raconte:

« J'étais scotché par l'influence de ces trucs-là : c'est quand il y avait le sondage de Telquel, un truc tout bête, j'ai fait, sur un Power Point, il y avait des templates toutes prêtes, où il y avait un petit oiseau qui gazouillait, qui disait quelque chose, j'avais qu'à enlever cette quelque chose et de mettre je suis un $9 \%$, je mets "je suis un $9 \%$ ", je me connecte deux ou trois heures plus tard et je trouve que tout le monde a mis cette photo-là sur la photo de profil, alors là, il y avait quelque chose ».

Un hashtag « \#9pcMaroc » (Figure 5) sur Twitter a également été utilisé. 
Figure 5. Tweets de « Ann `uss » (Anas Alaoui), initiateur du « Je suis un 9\%»

Par ailleurs, les blogueurs mobilisés dans cette campagne en ligne ont publié les résultats du sondage censuré. La mise en ligne de ce sondage peut être lue à la fois comme un geste de défi lancé aux autorités marocaines et comme une croyance dans une des promesses du web, à savoir sa capacité à " échapper » à tout contrôle étatique.

Les exemples présentés ci-dessus sont liés à des pratiques politiques ponctuelles, créatives, délimitées temporellement et qui peuvent se décliner sous différentes formes de formats numériques. Ces pratiques sont généralement individuelles, faits d'immigrés ou impliquant ces derniers dans leurs médiatisations, circulation et visibilité auprès d'un large public.

De telles pratiques politiques en ligne sont le plus souvent considérées par les internautes comme l'« arme » des dominés face au black- 'ut médiatique et aux injustices sociales. Elles donnent par ailleurs à voir la porosité des frontières entre les pratiques politiques des immigrés et celles des Marocains au Maroc. Les effets topologiques du web permettent ainsi à ces deux types d'acteurs de consulter les mêmes espaces pour s'informer et pour laisser des commentaires. Les immigrés peuvent être tantôt les initiateurs de ces mobilisations, tantôt le relais à la fois auprès d'un public national et international. La visibilité et la notoriété dont jouissent quelques blogueurs immigrés à l'échelle nationale marocaine et internationale leur permettent de participer à la médiatisation de ce type de pratiques.

$\mathrm{Si}$, dans le présent essai de genèse des pratiques politiques en ligne, un intérêt a été donné aux pratiques qui ont suscité une " mobilisation collective » et, de fait, ont fait connaître leur " existence » auprès d'un large public de Marocains, il n'en est pas de même pour les pratiques étudiées dans ce qui suit. En effet, il sera plutôt question de pratiques nous permettant de comprendre et d'interroger ce que sont les "pratiques politiques en ligne " d'immigrés marocains sans que celles-ci 
Quand des immigrés prennent la par'le. Appropriation des plateformes de $b /$ ' gging à des fins politiques par des immigrés marocains

ne soient impérativement liées à « des processus de consultation, de décision ou de mobilisation collective » pour reprendre le propos de Marcoccia (2003, p. 15).

\section{Les blogues comme espace pour faire et écrire la politique du pays d'origine}

\subsection{Point de vue méthodologique}

Notre corpus est constitué essentiellement des trois blogues suivants :

- http://www.larbi.org

- http://takhouar.wordpress.com

- http://sonofwords.blogspot.fr

II n'est pas représentatif des blogues politiques d'immigrés marocains, mais en est toutefois significatif. En effet, ce corpus donne à voir la diversité et l'hétérogénéité de ces espaces : on a ainsi affaire à un des blogues (Larbi) les plus lus et commentés de la " blogoma ${ }^{33}$ ", à un blogue politique collectif empruntant le registre de l'ironie dans son analyse et son écriture de l'actualité (Takh'uar) et au blogue d'un immigré installé en Amérique du Nord ${ }^{34}\left(S^{\prime} n\right.$ ' $\left.f w^{\prime} r d s\right)$. Toutefois, notre analyse s'est vue enrichie par des éléments issus d'autres blogues d'immigrés marocains, par des articles de presse et par des données circulant en ligne (sites web d'information, réseaux sociaux, etc.).

\footnotetext{
${ }^{33}$ Terme utilisé par les blogueurs et journalistes pour désigner la blogosphère marocaine (y compris les blogues des immigrés), toute catégorie de blogues confondue. Le terme $b l^{\prime} g$ ' ma suppose une homogénéité régnante au sein de ces blogues, à la fois du point de vue des acteurs sociaux, qui en sont les instigateurs, de ses catégories, etc. Ce terme pose la question de la pertinence de la transposition des frontières géographiques sur le web. Comment définir, dès lors, une blogosphère marocaine ou une « blogoma "? Comment délimiter les réalités qu'elle couvre ? Faut-il s'intéresser à la nationalité de ses blogueurs-acteurs, à la domiciliation des serveurs, au nom de domaine, aux thématiques abordées dans le blogue, à la langue de rédaction des billets, etc. ?

${ }_{34}$ Puisqu'un grand nombre d'immigrés marocains résident en France et en Europe, le nombre de blogues politiques d'immigrés résidant dans ces zones géographiques est plus élevé que celui des immigrés résidants dans d'autres contrées. Soulignons toutefois que l'Amérique du Nord optant pour une politique d'immigration sélective ou choisie, un grand nombre des immigrés marocains qui y résident disposent d'un capital culturel et économique élevé et s'approprient différentes plateformes web, entre autres, à des fins politiques.
} 
Créé par Larbi ${ }^{35}$, immigré marocain en France, un 30 octobre 2004, le blogue Larbi est l'un des plus connus de la blogosphère marocaine. Le blogue a été sélectionné deux fois, dans le cadre du concours Mar`c bl'gs Awards, comme le meilleur blogue de la catégorie "Social/politique ". Le titre du blogue a fait l'objet d'un changement permanent. À ses débuts, le blogue s'intitulait « Comme une bouteille jetée à la mer ! Blog d'un marocain résidant en France ${ }^{36}$ ", pour devenir "Choses vues, choses lues ॥ lors des mobilisations sociales de 2011 au Maroc $^{37}$. Les différents titres de ce blogue donnent à voir un imaginaire très récurrent au sein de la «blogosphère marocaine ", à savoir celui du caractère trivial et subsidiaire de la parole circulant au sein des blogues ${ }^{38}$.

Le blogue collectif Takh uar est créé en mai 2010 et intitulé CJDM, pour "Cercle des Jeunes Débiles marocains ». À travers ce titre, un avant-goût de l'univers satirique qui caractérise cet espace est proposé aux internautes lecteurs. Ce titre est loin d'être anodin. En effet, il propose un détournement du nom du mouvement «Cercle des Jeunes Démocrates Marocains ॥, mouvement affilié au Parti de l'Authenticité et de la Modernité (PAM). Ce parti, souvent désigné par ses détracteurs comme "le parti de l'ami du roi ", a fait l'objet de maintes critiques et dénonciations lors des manifestations du mouvement du 20 février, manifestations ayant eu un grand écho $^{39}$ au sein de la blogosphère. Ce blogue collectif est animé par cinq jeunes blogueurs marocains: Aboulaheb, jeune marocain résidant en France et de loin le plus actif du collectif, Lbadikhou, jeune marocain installé en Suisse, Khoroto, The wanderer, et Ze3boul. Le mot Takh 'uar figurant dans l'adresse URL du blogue est issu du dialecte marocain et signifie

\footnotetext{
${ }^{35}$ Le recours du blogueur à l'usage de son prénom dans le nom de domaine de deuxième niveau de l'adresse URL du blogue peut faire l'objet d'une double lecture. En effet, tout en garantissant un minimum d'anonymat au blogueur, l'usage du prénom instaure une proximité avec l'ensemble des internautes-lecteurs ou des commentateurs.

${ }^{36} \mathrm{Si}$ la phrase « Comme une bouteille jetée à la mer » a persisté dans le titre, la mention de la condition d'immigré du blogueur ou de sa double appartenance n'était pas constante. Toutefois, un clin d'œil à ce statut d'immigré se donne à voir à travers le rubriquage mis en place dans le blogue. Celui-ci évoque à la fois l'imaginaire des pays d'origine et d'installation, et ce, à travers les deux rubriques « Politique marocaine » et "Politique française ».

${ }^{37}$ Ces mobilisations ont été initiées par le mouvement du 20 février, mouvement ayant pris pour nom la date du premier jour de ces mobilisations sociales appelant à la dignité, la justice et la liberté.

${ }^{38}$ Ainsi, plusieurs titres de blogues donnent à voir cet imaginaire, comme c'est le cas du blogue de la blogueuse " une marocaine », intitulé " Mon agora : 7na ghadaouyine ", ce qui signifie « ce sont juste des paroles ».

${ }^{39}$ Ces manifestations ont été commentées, publicisées (annonces des manifestations et de leurs trajets) par les blogues qui les soutenaient.
} 
Quand des immigrés prennent la par 'le. Appropriation des plateformes de $b /$ ' gging à des fins politiques par des immigrés marocains

"baliverne ". On se retrouve encore une fois face à cet imaginaire de la "trivialité » des discours circulant au sein des blogues.

Enfin, le blogue $S^{\prime} n$ ' $f w^{`} r d s$ est créé en mars 2006 par Abmoul, pseudonyme d'un immigré marocain résidant au Canada. Ce blogue a connu une dynamique de publication et d'actualisation de son contenu dès le début des manifestations organisées dans le sillage du 20 février.

Faut-il encore souligner que les blogues d'immigrés, toutes catégories confondues, sont précurseurs de l'appropriation du web par les Marocains et, par conséquent, de l'investissement des espaces en ligne à des fins politiques, d'expression d'humeur et autres. En effet, ces immigrés ont bénéficié de la "généralisation » d'Internet dans leur pays d'installation, essentiellement des pays européens ou d'Amérique du nord, qui était antérieure à celle-ci au Maroc. En effet, la " compulsion de la proximité » (Diminescu, 2002) explique les différentes stratégies mises en place par les immigrés pour chasser les bonnes occasions afin de garder le contact avec le pays d'origine et, en l'occurrence, de suivre, de commenter et d'échanger autour de son actualité.

Le projet éditorial des blogues retenus dans cet article est politique ${ }^{40}$. Une richesse et une diversité des discours qui y circulent sont à noter. En effet, de nombreuses thématiques y sont abordées, allant de la situation des secteurs de la santé et de l'éducation au Maroc aux problèmes des libertés individuelles et de la presse, en passant par les questions d'injustice sociale, de corruption, etc. Outre leur caractère politique, force est de constater que la quasi-majorité de ces thématiques gravitent autour du pays d'origine ${ }^{41}$.

Le choix des thématiques abordées au sein de ces blogues n'est pas arbitraire. D'une part, un intérêt particulier est donné aux sujets estimés comme délaissés ou marginalisés par les médias officiels marocains muselés. Ces derniers sont accusés de proposer une couverture médiatique partiale et partielle des problèmes auxquels le Maroc fait face. Dans ces cas, ces blogues s'inscrivent en faux par rapport au traitement médiatique proposé par les médias officiels. Ils proposent et font circuler un discours politique relevant ainsi tantôt du registre de l'information, tantôt de celui du commentaire de l'actualité.

\footnotetext{
${ }^{40}$ Ils se distinguent par conséquent des blogues appartenant à d'autres catégories de blogues d'immigrés : blogues culinaires, d'humeur, de littérature, etc.

${ }^{41}$ Quelques billets portent toutefois sur l'actualité du pays d'installation, voire même sur l'actualité internationale. C'est le cas par exemple de ceux portant sur les élections françaises ou canadiennes.
} 
D'autre part, et étant donné que les blogues sont des dispositifs communicationnels où la dimension interactive est centrale, le choix des thématiques qui y sont abordées tient aussi compte des horizons d'attente des internautes-lecteurs. Un blogue ne peut exister sans ses lecteurs, qui s'approprient et actualisent les discours qui y circulent. Ainsi, tout un travail de sélection, combinant des critères de pertinence et d'audience, doit être mené en amont par le blogueur-scripteur. Un imaginaire du "bon blogueur " ${ }^{42}$ se dégageait ainsi des entretiens que nous avons pu conduire.

\subsection{De la question de la polyphonie à celle de la légitimité de l'instance énonciatrice des blogues}

La diversité des thématiques traitées croise celle des stratégies narratives mobilisées par les blogueurs. Ces stratégies, si elles sont informées par les architextes des blogues, changent également en fonction des pratiques créatives et des compétences rédactionnelles de chaque blogueur.

L'usage de la première personne du singulier est très fréquent dans ces espaces. D'aucuns peuvent considérer cette affirmation comme allant de $s^{\prime} i$, vu l'appartenance des blogues aux dispositifs d'autopublication. Ces dispositifs s'inscrivent dans la continuité de deux formes plus classiques que sont le journal intime et la lettre, où les formes d'expression des subjectivités sont courantes (Deseilligny, 2008).

Une autre lecture de l'usage fréquent du je est possible. Elle donne à voir l'expression d'individus revendiquant leur statut de citoyen agissant. C'est donc le citoyen, ayant des droits et des devoirs, qui s'exprime et qui expose publiquement ses points de vue sur les affaires de la Cité. En d'autres termes, le je est à la fois une négation de la figure du citoyen passif ${ }^{43}$ et une revendication de la figure du citoyen actif souhaitant partager sa parole et participer aux débats publics. L'usage du je,

\footnotetext{
${ }^{42}$ Ainsi, un des blogueurs que nous avons interviewé dégage « les règles d'or à respecter pour être un bon blogueur : 1) Être honnête et ne pas essayer de faire comme un autre blogueur qui a réussi ; 2) Être authentique : je sors mes propres tripes ; 3) Se spécialiser [...] ; 4) Être simple : ne pas se la jouer intello ; 5) Être généreux : c'est-à-dire ne pas être comme ces blogueurs qui ne donnent pas l'information gratuitement parce qu'ils veulent de l'argent. II faut bien se documenter sur le sujet sur lequel on va écrire, il faut prendre des photos ; 6) Ne pas être provocateur comme 7didane, le spécialiste de la blogosphère marocaine dans la provocation ».

${ }^{43}$ Cette passivité tend particulièrement à s'accentuer avec la distance liée à l'acte migratoire.
} 
Quand des immigrés prennent la par'le. Appropriation des plateformes de $b /$ ' gging à des fins politiques par des immigrés marocains

donnant à voir la subjectivité du citoyen-acteur, est encore davantage soutenu à travers la mobilisation de verbes modaux.

En parallèle à cet usage du pronom personnel je, ces blogueurs ont également recours au pronom $n^{\prime}$ us. Celui-ci marque l'adhésion et l'identification à une collectivité qui, le plus souvent, est nationale ${ }^{44}$, en l'occurrence la collectivité de Marocains ${ }^{45}$. Cet usage du $n$ 'us inscrit dès lors les blogueursscripteurs en position de porte-parole de la communauté nationale ${ }^{46}$. Ainsi, dans certains billets, ces blogues s'apparentent à des porte-voix parlant au nom de tous ceux qui ne disposent pas de compétences matérielles ou cognitives pour s'exprimer. Se pose alors la question de la légitimité de la parole politique circulant dans ces espaces et qui se donne à voir à deux échelles.

D'un côté, des internautes-commentateurs récusent le caractère représentatif de la parole de ces blogueurs. Ainsi, des commentaires d'internautes du type "ne parlez pas en mon nom " dénuent les billets de leurs valeurs symboliques. D'un autre côté, on dépossède les immigrés de leur droit de commenter ou de critiquer la vie politique du pays d'origine. Leur parole politique est considérée comme étant illégitime dès lors qu'elle est produite depuis une territorialité autre que celle du pays d'origine ${ }^{47}$. Pour d'aucuns, "être marocain " est intimement lié à un ancrage territorial. Or l'appropriation à des fins politiques des espaces en ligne par des acteurs

\footnotetext{
${ }^{44}$ Dans quelques cas précis, le $n$ `us fait référence à une communauté de pratique (groupe de blogueurs) ou à une communauté régionale. C'est particulièrement le cas de billets traitant du conflit israélo-palestinien. Le $n^{`}$ us fait référence dans ces billets à l' « identité arabe ».

${ }^{45}$ Un imaginaire du pays d'origine se donne à voir à travers la pratique d'écriture de ces blogues. Il y est désigné par une multitude de dénominations, telles que « le pays ", " chez nous » (faisant référence a l'appartenance à une territorialité autre que celle où se trouve le blogueur-auteur), " leplubopaysdumonde » (écrite en langage SMS et utilisée plutôt ironiquement) ou, enfin, par l'usage des déictiques comme « là-bas ».

46 «La Mauritanie, ce pays à nos frontières qu'on aime, nous autres Marocains, ignorer et pour certains, mépriser [...]. [Les Mauritaniens] viennent d'élire un parlement et un président qui les représentent » (billet extrait du blogue Sonofwords, 17 mars 2007).

${ }^{47}$ Ainsi lit-on dans un commentaire indexé à un billet du blogue Larbi portant sur le projet de la constitution marocaine de 2016 : « Tout d'abord, on n'attendait pas moins de toi... si tu crois nous faire une surprise avec ce genre de littérature, c'est raté, tu te trompes lourdement. [...] Attends donc d'y voir un peu clair dans ta langue maternelle le français et ses gauloiseries, puisque tu as besoin qu'on te traduise... on attendait de toi que tu traduises toi-même, pour ce qui nous concerne, à nous, mais à toutes autres compatriotes gaulois d'outre-mer, [...] tu as décidé de ne jamais être du côté de ton pays quoiqu'il fasse, et tu te donnes des airs de traiter, à la manière du crapaud de La Fontaine d'égal à égal avec le ROI ».
} 
déterritorialisés vient éclater cette limitation du « faire de la politique » à une seule géographie territoriale.

La légitimité d'un blogueur et, par conséquent, de son blogue se construit. Les stratégies analytiques, rédactionnelles et argumentatives données à voir dans les différents billets, ainsi que le choix des thématiques à traiter et leurs angles d'analyse participent à son alimentation.

Pour prendre l'exemple de Larbi, le blogueur fait appel aux chiffres et à l'imaginaire de la neutralité qui les entourent et met en exergue, dans un effort d'inventaire statistique, le nombre de billets et de commentaires publiés à la date de consultation du blogue (Figure 6). Le nombre de billets relativement élevé évoque l'effort d'écriture, d'actualisation et d'entretien du blogue, un effort qui ne relève pas des compétences de tout un chacun.

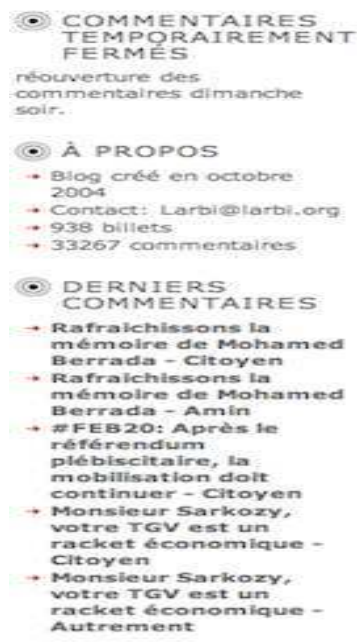

Figure 6. Éléments du paratexte et la construction de l'ethos du blogueur

La légitimité d'un blogue peut être aussi la conséquence de la médiatisation dont il fait l'objet. Plusieurs titres de presse se sont intéressés, ces dernières années, aux pratiques de bl'gging politique. Les blogues y sont présentés comme portant une nouvelle dynamique critique et incarnant un modèle alternatif à la presse officielle. Aussi, des blogues d'immigrés deviennent des sources d'information à part entière pour des titres de presse internationaux, comme Le C' urrier internati ' nal ou le journal britannique The Guardian ${ }^{48}$.

\footnotetext{
${ }^{48}$ À titre d'exemple, l'article « Why I reject Morocco's new constitution » (23 juin 2011) du blogueur Larbi
} 
Quand des immigrés prennent la par'le. Appropriation des plateformes de $b /$ ' gging à des fins politiques par des immigrés marocains

De même, l'éthos du blogueur se construit à travers les différents éléments du paratexte de son blogue, tels que le titre du blogue, le pseudonyme du blogueur, les causes soutenues, la $b l^{\prime} g r^{`} \|$, etc. L'ensemble de ces éléments participe à la construction d'une image du blogueur auprès de ses lecteurs dès leur première visite de cet espace en ligne.

Si l'on prend le cas des pseudonymes, ceux-ci ne sont pas fortuits. Ils font le plus souvent suite à des tactiques mises en place par les blogueurs. Tout en garantissant leur anonymat, ces pseudonymes reflètent des stratégies d'autodésignation de ces acteurs-blogueurs. On a ainsi affaire, entre autres pour le cas des blogueurs immigrés, à l'usage des seuls prénoms comme pseudonymes. Cet usage du prénom garantit à la fois un minimum de brouillage de l'identité du blogueur tout en la livrant en partie (c'est le cas, par exemple, du blogueur Larbi). Aussi, d'autres pseudonymes de blogueurs immigrés donnent à voir la revendication d'une identité nationale (comme pour la blogueuse " une marocaine »). Dans le même sens, d'autres blogueurs puisent dans le registre des personnages mythiques marocains, c'est le cas des deux blogueurs immigrés 7didane et Aisha Qandisha. D'autres encore puisent dans des répertoires à dimension davantage religieuse; le cas du blogueur Aboulahab ${ }^{49}$ est significatif à cet égard.

L'anonymat s'apparente, selon James Scott (2008), à une forme élémentaire de l'art du déguisement politique à travers lequel «les groupes dominés parviennent à insinuer leur résistance dans le texte public, sous des formes déguisées " (p. 153). Il devient, comme l'affirme Marco Marcoccia (2003), " une ressource stratégique pour les internautes. [II] favorise [d'une part] la capacité de produire des arguments, qui ne seront pas jugés uniquement sur la base de l'autorité de leurs auteurs $^{50}$ " (p. 44), et il permet aux internautes-blogueurs marocains, d'autre part, de s'exprimer plus librement sur des thématiques relevant de ce qu'Ahmed Hidass (2003) appelle "les exceptions fondamentales à la liberté d'expression ${ }^{51}$ "

(<https://www.theguardian.com/commentisfree/2011/jun/23/morocco-newconstitution>, dernière consultation le 18 mars 2017).

${ }^{49}$ Le choix du pseudonyme est loin d'être fortuit. Aboulahab (littéralement « père des flammes ॥) est l'un des oncles paternels du prophète Mohammed. II était l'un des farouches ennemis et opposants de ce dernier au point que la sourate $11 \mathrm{Al}$ Massad (Les fibres) du Coran l'a condamné nominativement.

${ }^{50}$ Aussi, et comme nous l'avons souligné, le paratexte des blogues participe à la construction de l'éthos du blogueur et, par conséquent, à son autorité au sein de la blogosphère.

${ }^{51}$ Par ces exceptions, il faut entendre ce qui est communément désigné les « lignes rouges » et qui englobent, en premier lieu, la personnalité du roi. Celle-ci ne peut faire l'objet ni de propos de lèse-majesté ni de propos irrévérencieux. En second lieu 
(p. 258). Ainsi, le brouillage auctorial autorise des blogueurs à se pencher sur ces thématiques, considérées autrement comme étant critiques.

Toutefois, et pour beaucoup de blogueurs, cet art de résistance (Scott, 2008) n'est pas à lui seul caution d'une prise de parole libre et détachée de tout désagrément potentiel des autorités marocaines. L'ancrage géographique du blogueur est déterminant pour la définition d'une marge de critique à adopter. Ainsi, la blogueuse "une marocaine » précise, lors d'un entretien: "Le ton que j'adopte pour critiquer Mohammed VI, je ne peux pas l'adopter au Maroc ». L'écriture critique de la politique serait ainsi intimement liée à une question d'ordre géographique, à la territorialité. On ne blogue pas la politique marocaine de la même manière selon qu'on est à Casablanca ou à Paris.

L'anonymat, et essentiellement à travers sa forme élémentaire qu'est le pseudonyme, garantit un minimum de sécurité pour les blogueurs et pour les membres de leur famille restés au Maroc. Vu les multiples cas de prisonniers d'opinions au Maroc ${ }^{52}$, l'anonymat devient, selon le blogueur Hisham Al Miraat, une soupape de sécurité. Et il poursuit lors de notre entretien: " tous ces blogueurs ont leur famille au Maroc, une critique du régime peut leur causer "sda3" [des ennuis] ".

Cette tactique ne fait toutefois pas l'unanimité auprès de l'ensemble des blogueurs immigrés. Certains, comme Anas Alaoui et Reda Chraibi, s'inscrivent davantage dans une logique de dévoilement identitaire.

\subsection{De quelques stratégies narratives mises en œuvre dans ces espaces}

Du fait même de leur inscription en faux par rapport aux médias officiels marocains, l'argumentation devient un enjeu stratégique dans ces blogues. En effet, la rhétorique de la rationalité est souvent déployée dans ces espaces. Les blogueurs s'adressent ainsi à travers leurs billets à des citoyens ayant besoin de preuves et d'arguments pour être convaincus. Les sources d'information mobilisées sont donc toujours signalées dans un effort de documentation. On n'hésite pas à faire appel, entre autres, aux rapports d'ONG internationales ou

viennent la forme du régime (la monarchie), la religion d'État (l'islam) et la question du Sahara.

${ }^{52}$ Voir le rapport de Reporters sans frontières de 2016 :

$<$ https://rsf.org/fr/maroc?gclid=CjwKEAjw-r27BRCzsNyzrMrM-

j0SJACp2TiBJvmWiS8WZUtpp8gJGYoLF3W-zyJXAtv9yZ5F3fAm6BoCU3Hw_wcB>, dernière consultation le 18 mars 2017. 
Quand des immigrés prennent la par'le. Appropriation des plateformes de $b /$ ' gging à des fins politiques par des immigrés marocains

à des articles de lois pouvant servir d'arguments d'autorité ${ }^{53}$ (Figure 7).

Par ailleurs, les billets des blogues peuvent être considérés

Souveraineté, Paix et Justice (3) + Bibliographie

Justice et Souveraineté

\begin{abstract}
La justice en relations internationales est elle aussi en relation dialectique avec l'ordre et la stabilité. Il est difficile dans les relations entre États, comme c'est d'ailleurs le cas en politique domestique, de concevoir la justice sans l'ordre (Bull 1977). Or, l'ordre international, avec les règles et les institutions qui le soutiennent[i], peut être une entrave à la réalisation de certaines formes de justice. La souveraineté, mécanisme de réforme censé à l'interne approcher les principes de justice morale, peut aussi être source et cause d'injustice à l'échelle domestique et internationale. Ce paradoxe, relevé par Kant dans son traité de réforme internationale (Franceschet 2002), a subi les effets de l'évolution historique et des changements qu'on connues les communautés sociales et leurs relations mutuelles.
\end{abstract}

Qu'est-ce que la justice internationale?

\begin{abstract}
Nous avons exposé comment la conception de la paix a évolué dépassant sa teneur "négative" pour intégrer une dimension "positive" se référant au bien-être de la population, son accès aux besoins de base et la nécessité d'une solidarité internationale. De fait, la justice, ce concept abstrait comme le sont ceux de souveraineté et de paix, devrait aussi, pour acquérir une existence concrète et se dépouiller de sa polysémie, être confronté au monde phénoménal et séparée de la vertu. Hedley Bull (1977) a bien mis en évidence cette profusion de sens dépendamment du niveau d'analyse et de la propre subjectivité de ceux qui en parlent[ii]. De manière générale, à l'échelle des relations entre États, la justice internationale se référait aux "moral rules held to confer rights and duties upon states and nations" (Bull 1977). La souveraineté, et ce qu'il en découle comme droits à l'intégrité territoriale et à l'unité nationale, faisait (et fait toujours, du moins formellement) partie de ces droits, mais n'o hictorinmamant ma nan rócictá qux réalités des interactions au sein de la société
\end{abstract}

Figure 7. Billet publié dans le blogue Son of words montrant l'effort d'argumentation du blogueur-auteur. Billet publié le 23 janvier 2009.

comme des "récits déclencheurs » (Dupret, Klaus et Ghazzal, 2010) si l'on se fie aux réactions qu'ils suscitent dans l'espace commentaire. C'est le billet qui, par la médiation du récit qu'il met en scène, par son énonciation et par son argumentation, cadre les commentaires qui lui sont indexés. Dès leur titre, certains billets des blogues de notre corpus mettent en scène les protagonistes ou les actants du récit. Ces derniers, se trouvant en situation de conflit, sont quasiment tous situés sur l'axe « bon-mauvais » et associés à une axiologie morale.

\footnotetext{
${ }^{53}$ Voir aussi à titre d'exemple, ce billet du blogue Takh ' uar intitulé « Financements étrangers : le ministère de l'intérieur menace de diss ' udre la p` lice » daté du 11 janvier 2015 : https://takhouar. wordpress.com/2015/01/11/financements-etrangersle-ministere-de-linterieur-menace-de-dissoudre-la-police/
} 
Prenons l'exemple du titre du billet daté du 12 septembre 2011 du blogue Larbi, à savoir « Une Audi A8 pour Moncef Belkheyat, une cellule de prison pour Mouad L7a9ed » (Figure 8).

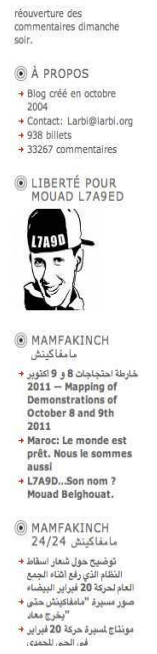

Une Audi A8 pour Moncef Belkhayat, Une cellule de prison pour Mouad L7aged
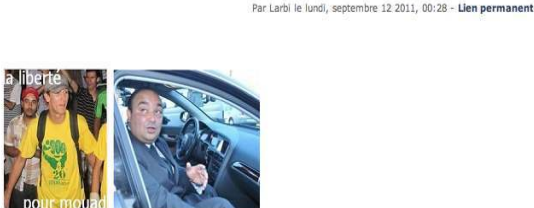

Le Maroc est un pays plein de corrompus et de vorts

La bonne nouvelle de la semaine c'est la voiture de Moncef Belkhayat qui ne coùte au contribuable marocain \& que *37000 Dhs par la luxueuse berline du ministre ne dégraisse le budget de IEtat que de $18 \mathrm{Smigs}$ par mois. Et encore on ne prend que le chiffrage du ministère. Ça ne doit pas être si choquant que ça vu que le ministre l'exhibe fièrement sur son site sur le mode : ma berline de luxe vaut bien le salaire de 18 prolétaires, par mois s'll vous plait. Un des courtisans du ministre ose justifier \& Belkhayat n'a pas attendu standing à la hauteur de son ill bstre personne.

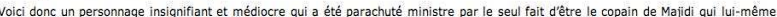
est copain du Roi, et qui toute honte bue, se croit autorisé en ces temps durs à réaliser son orgasme, rouler en une Audit 8 , sur le dos du contribuable. Qui plus est, a linsolence de répondre, pour sa défense, que * disposer d'une voiture de fonction est un droit

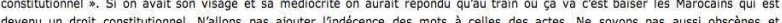

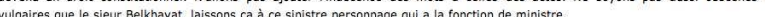

Figure 8. Capture d'écran du blogue Larbi. org

II est question, dans ce billet, de deux actants: Moncef Belkheyat, ancien ministre des Sports et de la Jeunesse marocaine, et Mouad L7a9ed ${ }^{54}$, rappeur marocain " engagé ». Les deux actants se trouvent en situation d'opposition, qui s'accentue encore davantage avec l'usage de deux images qui accompagnent le billet: l'une présentant Belkheyat, souriant dans sa voiture, et l'autre présentant L7a9ed, littéralement « le rancunier ", scandant des slogans en pleine manifestation. Tout oppose les univers des deux actants, dont le premier, représentant la figure du "méchant », est mêlé à une affaire d'abus de pouvoir et le second, représentant le « héros militant », est incarcéré. La dialectique existante entre ces deux images et le texte peut être parfaitement explicitée à travers cette citation de Philippe Marion (1997) :

«entre l'image et l'écrit se joue une construction herméneutique, un fragment de mise en intrigue. Les éléments montrés par l'image sont relayés par le texte qui développe à son tour des mécanismes de dramatisation destinés à être eux-mêmes réinjectés dans l'interprétation de l'image » (p. 84).

${ }^{54}$ Le « 7 » fait appel à la lettre $\mathrm{H}$ et le « 9 », à la lettre $Q$. 
Quand des immigrés prennent la par'le. Appropriation des plateformes de $b /$ ' gging à des fins politiques par des immigrés marocains

Et le blogueur-scripteur, Larbi, poursuit: "Le Maroc est un pays plein de corrompus et de voyous et ce sont les "affreux" militants pacifistes qu'on met en prison... La voilà l'exception marocaine ". La critique du pouvoir en place ne se fait pas uniquement à travers des discours abstraits et qui généralisent ; on cible au contraire des personnalités politiques bien précises, le plus souvent preuves à l'appui. Un vrai travail d'enquête et d'investigation est conduit pour dénoncer certaines exactions et abus du pouvoir (Figure 9).

\section{Oligarchie}

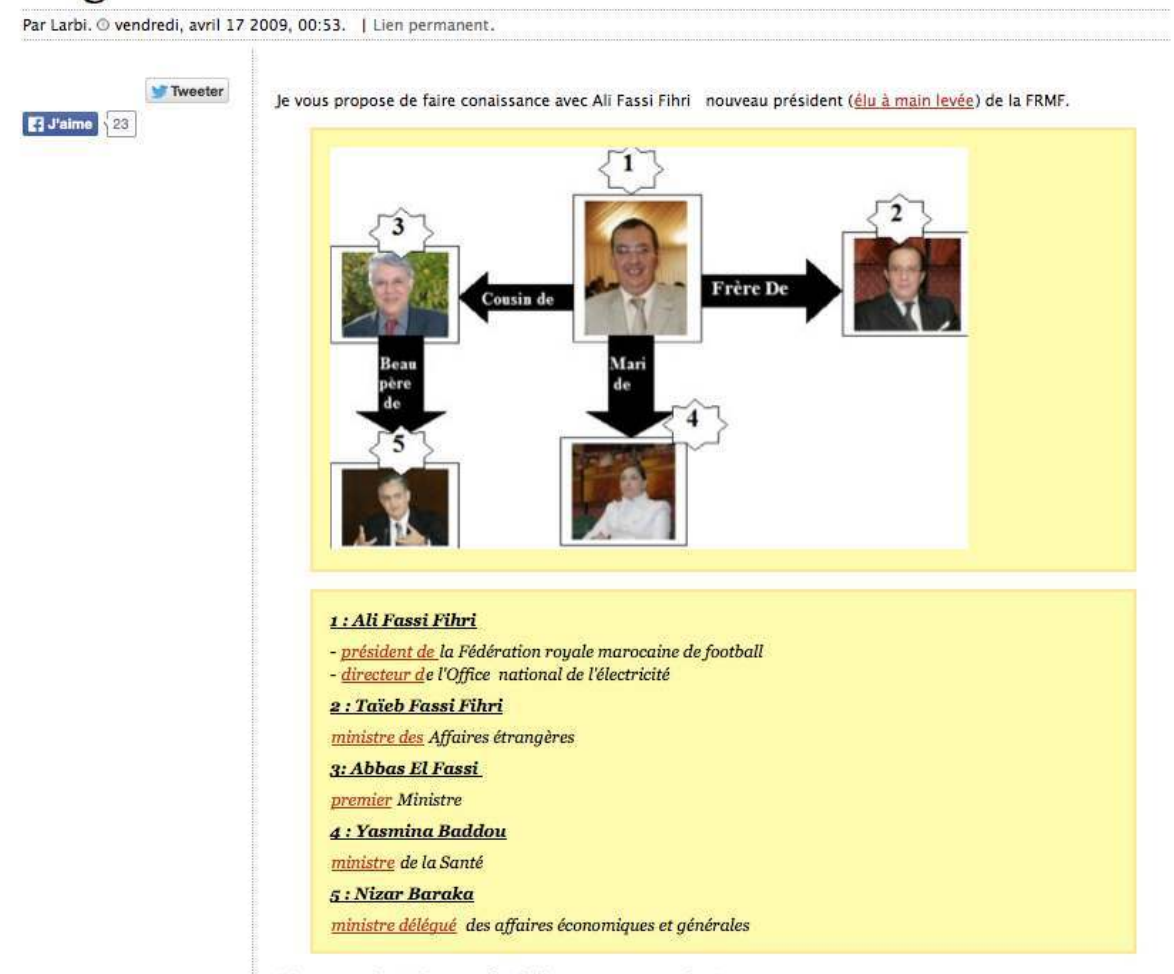

Figure 9. Billet daté du 17 avril 2009 dénonçant « l'oligarchie au Maroc » (<http://www.larbi.org/post/2009/04/Oligarchie>)

Par ailleurs, de nouvelles formes d'écriture de la politique fortement inspirées du répertoire d'actions collectives hors ligne se donnent à voir à travers les blogues. On n'hésite pas à faire appel, par exemple, aux "lettres ouvertes". Ce trope communicationnel $^{55}$ (Kerbrat-Orecchioni, 1994) est très souvent utilisé dans la presse où l'auteur de la lettre feint de s'adresser

\footnotetext{
55 «[C]onsiste à feindre d'adresser à A1 un énoncé qui est en réalité adressé à A2 : on tente par ce subterfuge, en ne lui disant pas les choses "en face", de ménager celle de son véritable destinataire » (Kerbrat-Orecchioni, 1994, p. 68).
} 
à un destinataire alors qu'il s'adresse à tous ses lecteurs. Quand elles sont mobilisées dans ces blogues, les lettres sont rédigées généralement à l'intention de personnalités politiques et publiques à des fins dénonciatrices et critiques ${ }^{56}$.

Un autre exemple de ces pratiques créatives d'écriture de la politique est le blogue Takh uar. Par son titre, ce blogue submerge l'internaute-lecteur dans son style satirique et ironique. Rares sont les titres de presse au Maroc qui ont exploité ce choix stylistique. Les quelques expériences journalistiques qui ont vu le jour ont été censurées et vouées au silence (à titre d'exemple D' umane, Demain). L'ironie permet d'aborder des sujets considérés comme tabous avec humour et satire, mais pour atteindre son but, elle nécessite des compétences linguistiques à la fois de la part du blogueurscripteur et de l'internaute-lecteur, qui doit saisir le sens des antiphrases. On trouve, à titre d'exemple dans le blogue Takh'uar, un dictionnaire intitulé The OXI'I Dicti' nnary ' $f$ $M ` r$ ccan $p$ 'litics, qui englobe un certain nombre d'entrées proposant des définitions, non sans humour et ironie, sur la vie politique marocaine. La lettre $X$ y est par exemple définie comme " lettre de l'alphabet, [qui] se dit de la direction où va le Maroc ». La bl' gr' II s'inscrit également dans le sillage de cet univers satirique. Elle comprend trois signes passeurs intitulés " Nouveau testament ", "Ancien testament » et "Bible », qui orientent l'internaute-lecteur, consécutivement, vers les sites web de journaux officiels et de l'agence de presse marocaine, connus pour être sous le joug direct du pouvoir, à savoir respectivement Auj' urd'hui le Mar'c, le Matin et la MAP (Maghreb Arab Press).

\subsection{L'espace commentaire et sa polarisation: entre échange et conflit}

Compte tenu de leur dimension interactive, on se trouve face à deux registres de communication au sein de ces blogues, à savoir le billet et les commentaires produits par deux instances énonciatrices différentes. La disposition visuelle de l'espace commentaire à la suite de chaque billet associe ainsi le geste de commenter au geste préalable de lecture.

Une polyphonie de l'instance énonciatrice est à l'œuvre dans les blogues. La voix du blogueur-scripteur n'est pas la seule qui

\footnotetext{
${ }^{56}$ Comme c'est le cas de cette lettre ouverte adressée à l'ancien ministre de la Communication, Khalid Naciri, dans le blogue Takhouar et intitulée « Monsieur le ministre, vous êtes un génie »

(<https://takhouar.wordpress.com/2010/11/24/monsieur-le-ministre-vous-etes-ungenie/>, dernière consultation le 26 mars 2016).
} 
Quand des immigrés prennent la par'le. Appropriation des plateformes de $b /$ ' gging à des fins politiques par des immigrés marocains

y circule. Celle des internautes-commentateurs l'accompagne. De fait, et comme le précise Michel Marcoccia (2003), l'espace dédié aux commentaires rend les blogues semblables à une discussion toujours en cours, un "état de parole ouvert». L'internaute-lecteur est loin d'être passif. Sa part créatrice, qui se donne à voir à travers les commentaires, l'institue comme acteur de la coconstruction du récit du billet ${ }^{5}$.

Les commentaires viennent tantôt corroborer et appuyer les propos tenus dans le billet, tantôt les nuancer ou les critiquer. Les commentaires peuvent aussi s'apparenter à de vraies tactiques des internautes. Certains n'hésitent pas à publier dans cesdits espaces des messages d'autopromotion de leurs propres blogues $^{58}$, des annonces de pétitions à signer, etc.

Un brouillage auctorial des internautes-commentateurs est à noter au sein de ces espaces. Un large éventail de pseudonymes est mobilisé, et qui n'est pas dénué, pour certains, de significations, de références et de revendications identitaires. On a ainsi affaire à des pseudonymes faisant référence à de simples prénoms ("Ahmed ", "Adil », etc.), à une qualification géographique ("Ahmed Rifi»), à une ville ( "Nadori de chez Nador»), à une appartenance nationale ("Marocain, Pur Marocain », etc.), à des qualifications religieuses («Musulman», etc.), etc. Le choix des pseudonymes découle souvent de la thématique abordée dans le billet, mais aussi des échanges menés dans l'espace commentaire. Précisons que ces internautes-commentateurs s'ancrent dans différentes territorialités. Autrement dit, ces commentaires sont à la fois des productions discursives d'internautes immigrés et d'internautes au Maroc.

L'usage de l'anonymat à travers sa forme élémentaire que sont les pseudonymes n'est pas sans dérives. Si cette tactique permet aux internautes-commentateurs de commenter plus "librement » et d'adopter un ton direct et critique, elle peut être aussi défaillante et manquer à l'idéal d'une discussion responsable et rationnelle. II est aisé de repérer au sein de l'espace commentaire d'un blogue des traces de conflits entre les différentes instances énonciatrices participant à la discussion. Ces conflits prennent souvent la forme d'insultes ou de diatribes (Danet, 1998), ainsi que des injonctions ou remises

\footnotetext{
${ }^{57}$ Mentionnons toutefois que le nombre de commentaires indexés aux billets varie au sein d'un même blogue et d'un blogue à un autre. En effet, certains billets génèrent un grand nombre de commentaires publiés, et ce, selon la thématique analysée ainsi que le positionnement qui y est tenu par le blogueur. Par ailleurs, et étant donné l'éthos de leur blogueur ainsi que de leur visibilité sur le web, certains blogues génèrent plus de commentaires que d'autres.

${ }^{58}$ Nous avons constaté que plus la visibilité du blogue et sa notoriété sont grandes, plus le nombre de ce type de messages est élevé.
} 
en ordre de la part d'autres protagonistes du débat. De plus, la parole déguisée devient encore plus suspecte dans le cadre d'un État en transition démocratique, où elle est assimilée, dans certains cas, à la présence d'une cyberpolice. En effet, si la surveillance officielle du réseau des réseaux peut se faire à travers un arsenal de dispositif juridique, elle peut également se produire à l'intérieur même de ces espaces web.

On peut distinguer deux types de surveillance depuis les interstices du web: une surveillance menée par une cyberpolice marocaine et une surveillance conduite par les "fidèles du Makhzen ", pour reprendre l'expression de Abdelfettah Benchenna (2011), c'est-à-dire ces internautes défenseurs de la politique officielle conduite au Royaume et ces défenseurs du monarque d'une façon plus générale (Figure 10). En effet, une cyberpolice marocaine, à laquelle le législateur fait allusion dans l'article 77 du code du numérique, existe depuis 10 ans sous l'appellation officielle de "police technique ${ }^{59}$ ". La date de naissance de cette cyberpolice croise le début de la "généralisation " d'Internet au Maroc et donc du besoin naissant de contrôler cet espace, du moins de le surveiller lui ainsi que les contenus qui y circulent.

\begin{abstract}
3. Lâcher les baltajia[1] du net
Des groupes se créent sur facebook et autres réseaux sociaux appelant à la révolte. Pas de panic. Créer des groupes rivaux mettant en garde contre le chaos, les ennemis de la nation, etc. Les usual suspects en bref. Les baltajia du net doivent intimider les cyber-dissidents, les menacer, les ridiculiser, pirater leurs comptes, etc. Éviter cependant de créer des groupes pour "la marche de l'amour" (c'est trop ridicule) ou une page de fans du dictateur (les services secrets du dictateur n'ont pas assez de ressources pour mobiliser tous leurs éléments cliquer sur fan de; et de toute façon, les espions du dictateur sont des ignares es internet comparés à ces diables de jeunes du net). Après l'écrasement de la révolution, le dictateur pourra réfléchir à tête reposée au traitement à réserver à l’imbécile qui a le premier pensé à introduire internet sur le sol de son pays.
\end{abstract}

Figure 10. Billet abordant la question de la cybersurveillance, par $S^{\prime} n^{\prime}$ ' $f w^{\prime} r d s$, "Petit guide du dictateur arabe en situation de crise révolutionnaire » (21 février 2011)

II demeure toutefois très difficile de distinguer les pratiques politiques en ligne s'inscrivant dans le cadre d'une stratégie étatique, en l'occurrence les pratiques de la cyberpolice, de

\footnotetext{
${ }^{59}$ On lit, dans cet article : « Dans le but de constater les infractions commises par un moyen de communication numérique en ligne, d'en rassembler les preuves et d'en rechercher les auteurs, les officiers de police judiciaire et les agents de l'administration spécialement habilités agissant dans le cadre d'une procédure pénale peuvent procéder aux actes suivants sans en être pénalement responsables : participer sous un pseudonyme aux échanges électroniques ; être en contact par ce moyen avec les personnes susceptibles d'être les auteurs de ces infractions ; extraire, acquérir ou conserver par ce moyen les éléments de preuve et les données sur les personnes susceptibles d'être les auteurs de ces infractions. »
} 
Quand des immigrés prennent la par'le. Appropriation des plateformes de $b /$ ' gging à des fins politiques par des immigrés marocains

celles développées par des citoyens, en l'occurrence des « fidèles du Makhzen ». Leurs détracteurs n'hésitent pas à les désigner en tant que «milices numériques du roi Mohammed VI », ou comme l'« Armée invisible du web ». Ces " fidèles du Makhzen » s'autodésignent, dans différentes pages Facebook, en tant que "Mouvement des jeunes royalistes ", ou «Alliance royaliste marocaine ${ }^{60}$ ". Ils s'autoproclament comme représentants légitimes des « vrais " Marocains et affichent leur allégeance au roi. De ce fait, certains de leurs détracteurs les qualifient, comme nous le déclare dans un entretien le blogueur Hisham Al Miraat, de citoyens avec « un excès de zèle ", tandis que d'autres les décrivent comme " plus royalistes que le roi ».

Le conflit régnant au sein des espaces commentaires de ces blogues politiques croise la polarisation des blogueurs entre deux principales catégories, celle des " nihilistes » et celle des "makhzénistes ${ }^{61}$ ". Ces deux catégories s'opposent et se révèlent être, si l'on veut puiser dans le vocabulaire de l'analyse conversationnelle, une paire relati nnelle disj nctive. Cette opposition reflète ainsi les tensions et les conflits ayant lieu " hors ligne » entre les protagonistes de la politique officielle et ses détracteurs.

La catégorie des "nihilistes" regroupe l'ensemble des blogueurs et des commentateurs critiques, pour ne pas dire hostiles, au pouvoir politique en place au Maroc. Si cette dénomination correspond à une autodésignation des membres de cette catégorie d'appartenance, elle n'est en fait que le renversement d'un stigmate accolé à ces internautes. Ainsi, Larbi précise dans la rubrique "L'interrogatoire » du magazine Telquel: " Je n'ai jamais inventé ce terme puisqu'il a été utilisé à plusieurs reprises par les officiels du pays. Dans leur bouche, nihiliste devient une insulte. Mais moi, au lieu de m'en offusquer, j'ai décidé de le revendiquer haut et fort, comme un acte de résistance ॥. D'autres blogueurs revendiquent cette catégorie d'appartenance par le titre même de leur blogue. C'est le cas, par exemple, du blogue intitulé Nihilisme sanguinaire dont le sous-titre est " '[...] s'évertuer à semer le scepticisme et le nihilisme" Extrait d'une insulte $r^{\prime}$ yale ». L'autre catégorie d'appartenance se situant à l'antipode de la première est celle des "Makhzénistes ». Cette dénomination, ou plutôt cette assignation identitaire formulée par la première catégorie, regroupe l'ensemble des blogueurs et des

\footnotetext{
$60<$ https://www.facebook.com/mouvement.royalistes/> et <https://www.facebook.com/groups/greatmorocco1200/?fref=ts>, dernière consultation le 20 mars 2016.

${ }^{61}$ L'usage des guillemets est important puisqu'on reprend ici les dénominations utilisées par les blogueurs immigrés.
} 
commentateurs soupçonnés par les premiers d'être des "fidèles du Makhzen », voire des membres de la cyberpolice marocaine.

La pratique des commentaires semble toutefois être majoritairement une " affaire » d'habitués. La quasi-majorité des commentaires publiés dans ces blogues se révèlent être le fait des mêmes internautes signant leurs contributions avec les mêmes pseudonymes. Ces internautes-commentateurs « réguliers » sont majoritairement des auteurs-éditeurs d'autres blogues, installés au Maroc ou à l'étranger. Ce constat corrobore la métaphore de Sophie Pène (2007) selon laquelle les blogues s'apparentent à des petits mondes interconnectés. Sans leur inscription dans un ou plusieurs réseaux, un blogue ne peut avoir de visibilité sur le web.

\section{Qui blogue? Tentative de limitation des profils des blogueurs.}

Le réseau d'interconnaissance entre ces blogueurs immigrés se donne à voir à travers le développement de différentes pratiques hors ligne. Comme les blogueurs Hisham Al Miraat et Anas Alaoui nous l'ont déclaré, des rencontres sont régulièrement organisées par ces blogueurs et sont baptisées les " rencontres des couscousistes-tajinistes ${ }^{62}$ ". Le nom même de ces rencontres puise dans un référentiel culinaire qui fait partie intégrante de l'identité nationale marocaine. En dehors du cadre de ces rencontres susmentionnées, des échanges et d'autres contacts entre ces blogueurs ont, comme nous avons pu l'observer, eu lieu lors de manifestations culturelles ou de mobilisations politiques comme celles organisées en marge du mouvement du 20 février.

Une proximité, pour ne pas dire une homogénéité idéologique, entre ces différents blogueurs se donne à voir. Cette proximité affichée correspond, comme l'affirme Peter Dahlgren (2012), à un "schéma de comportement humain compréhensible : on évite les conflits et renforce les visions du monde et ses valeurs ॥ (p. 21). Cette tendance à se regrouper entre internautes qui partagent plus ou moins les mêmes visions du monde semble engendrer la construction de ce qui est désigné dans de nombreux travaux par l'expression " chambres d'écho ». Par cela, il faut penser à la « tendance des personnes à se regrouper à l'intérieur de réseaux par communautés d'opinions " (Dahlgren, 2012, p. 21). L'existence de ces "chambres d'écho » n'est pas uniquement

\footnotetext{
${ }^{62}$ La rencontre et l'échange en ligne entre ces blogueurs préexistent la rencontre physique hors ligne.
} 
Quand des immigrés prennent la par'le. Appropriation des plateformes de $b /$ ' gging à des fins politiques par des immigrés marocains

caractéristique des blogues. Elle est également visible sur d'autres plateformes, telles que Twitter et Facebook, et suscite par conséquent une polarisation à l'intérieur du «web marocain $"$.

Cette polarisation qui entrave la rencontre et l'échange rationnel entre les protagonistes d'avis divergents est renforcée par la pluralité linguistique des productions discursives sur ce web. La quasi-totalité des billets publiés dans les blogues étudiés dans ce travail est rédigée en langue française. Rares sont ceux écrits en langue arabe. L'usage de celle-ci s'inscrit dans le cadre de la pratique du $c^{\prime}$ de switching (alternance codique), définie par Louis-Jean Calvet (1993) comme "le changement de langue [qui] se produit dans le cours d'une même phrase ou d'une phrase à l'autre " (p. 22). En l'occurrence, l'arabe dans sa forme dialectale, est utilisé pour des expressions bien spécifiques telles que l'invocation de Dieu, la citation de proverbes, etc., bref tout ce qui est estimé comme non traduisible par les blogueurs ou perdant de sa charge symbolique et de sa force de sens.

La fragmentation du « web marocain » en petites bulles, dont la "langue " est l'un des facteurs structurants, entrave par conséquent l'interaction entre, par exemple, les blogues dont les productions discursives sont en français et ceux où elles sont en arabe.

La lecture, la compréhension et l'éventuel commentaire des billets nécessitent, dans le cadre du corpus étudié, un minimum de maîtrise de la langue française de la part des internauteslecteurs. Eu égard au taux d'analphabétisme et de pénétration d'Internet au Maroc ${ }^{63}$ ainsi qu'au nombre réduit de blogues politiques par rapport aux autres catégories de blogues, il s'avère que ces blogues politiques francophones sont minoritaires et ne touchent qu'un cercle fermé d'internautes.

En puisant à la fois dans les entretiens conduits dans le cadre de cette recherche, dans les discours d'escorte qui accompagnent certains blogues ainsi que dans la médiatisation dont bénéficient certains blogueurs, la figure des acteurs sociaux concernés par ce type de pratiques en ligne semble se dessiner. En effet, ces derniers sont principalement des jeunes

\footnotetext{
${ }^{63}$ Très peu d'études portent sur les pratiques médiatiques des immigrés marocains d'une façon générale et sur leurs pratiques en ligne plus spécifiquement. On cite à titre d'exemples les travaux d'Abdelfettah Benchenna (Benchenna, 2014), de Laura Navarro (Navarro, 2014), de Fathallah Daghmi (Daghmi, 2014) et de Siham Touhami (Touhami, 2007). Ainsi, il nous est difficile de communiquer des données chiffrées, précises et fiables quant à ces pratiques.
} 
hommes ${ }^{64}$ disposant d'un capital social et culturel important et résidant principalement en Europe et en Amérique du Nord. On a ainsi affaire à des jeunes cadres supérieurs (médecins, ingénieurs, informaticiens, consultants, juristes, professeurs, etc.) ayant fait toute ou une partie de leur scolarité, en l'occurrence leurs études supérieures, à l'étranger.

Ainsi, à l'opposé des discours qui prophétisent le caractère " ordinaire " des acteurs sociaux développant des pratiques politiques en ligne, on se retrouve en présence d'une élite occidentalisée. Cette dernière déclare n'afficher aucune appartenance partisane, mais des sympathies revendiquées par rapport à certains courants politiques. Ils n'hésitent ainsi pas à se définir dans les entretiens en tant que " progressistes » et " humanistes ». Ces blogueurs disposent aussi de ressources cognitives et temporelles nécessaires pour la création et l'animation d'un blogue. Ces deux ressources sont inégalement partagées parmi les rangs des internautes. Ce ne sont pas tous les internautes qui disposent du temps nécessaire ou de la volonté de s'investir dans l'animation d'un blogue, ou du "répertoire d'arguments " (Cappella, Price et Nir, 2002) requis pour une analyse critique et argumentée d'un évènement donné.

Le web étant un espace textuel, tous les échanges et les prises de parole qui s'y déroulent se font à travers du texte. Comme le soulignait Pierre Bourdieu (1997) dans son ouvrage Faç ' ns de parler, " les conditions de félicité " sont inégalement réparties. Écrire un discours logique, structuré et argumenté ne relève pas des compétences de chaque internaute. Ces derniers n'en sont pas pour autant dupes et adaptent ainsi leurs prises de parole à leurs compétences et ressources cognitives (certains choisissent par exemple d'utiliser les vidéos, d'autres de tenir des blogues, etc.).

\section{Conclusion}

Les pratiques politiques étudiées s'apparentent à des productions discursives créatives ou, en d'autres termes, à des pratiques d'écriture et de réécriture de la politique. Pourquoi des immigrés se livrent-ils à ce geste d'écriture du pays d'origine ? Pourquoi s'intéressent-ils et commentent-ils les affaires de la Cité où ils ne résident plus ?

Les immigrés investissent l'écriture d'une fonction réparatrice. À travers le geste d'écriture, ils entretiennent un lien avec le pays d'origine et maintiennent leur mémoire

\footnotetext{
${ }^{64} \mathrm{Peu}$ de femmes sont concernées par les pratiques du bl`gging et encore moins par le $b /$ ' gging politique.
} 
Quand des immigrés prennent la par' le. Appropriation des plateformes de $b /$ ' gging à des fins politiques par des immigrés marocains

collective. La distance géographique leur doterait même d'une réflexivité afin d'analyser ou de commenter l'actualité marocaine, car penser et écrire le pays d'origine renvoie à l'ancrage territorial de l'auteur-énonciateur. On n'écrit pas sur le pays d'origine, et en l'occurrence sur sa politique, de la même façon quand on y est ou quand on est à l'extérieur. Comme l'avance Del Rio Luis (1996), il faut prendre en compte "l'évolution de la structure mentale et sociale» (p. 9) de l'immigré.

Par ailleurs, l'écriture est considérée par l'ensemble des immigrés que nous avons interviewé comme une écriture catharsis, comme une " psychothérapie ${ }^{65}$ " permettant à la fois de mieux se connaître soi-même et d'accéder à un apaisement intérieur. D'aucuns n'ont pas hésité à préciser, dans nos entretiens, que cette écriture est d'abord "destinée à euxmêmes " avant de s'adresser aux Autres, aux internauteslecteurs.

Ces pratiques de faire de la politique en ligne menées par des acteurs exclus des voies classiques de participation politique leur octroient une visibilité auprès de leurs pairs immigrés et au Maroc. Ces pratiques seraient ainsi des reconquêtes d'une parole confisquée, aussi relatives et marginales soient-elles. Elles démontrent le refus de certains immigrés de rester cantonnés dans le statut de "citoyens de l'étranger ", autrement dit de "citoyens de seconde zone ", soumis au pouvoir étatique sans pouvoir y participer. Faut-il rappeler qu'il n'est pas, ici, question d'étudier un quelconque impact de ces pratiques politiques sur le processus de prise de décision au Maroc. En effet, dans ce travail, ces pratiques sont essentiellement considérées comme étant des acti $n s$ symb'liques. De plus, et d'après leurs déclarations, ces blogueurs-énonciateurs ${ }^{66}$ ne s'intéresseraient pas à la rec'nnaissance de leurs voix: leurs circulations et leurs appropriations sont ce qui importe le plus.

Michel de Certeau (1994) avance, sur ce double jeu de la parole politique se positionnant entre sa fonction fortement

\footnotetext{
${ }^{65}$ Dans ce sens, Hisham Al Miraat déclare : « Le bl’ gging est une sorte de psychothérapie. II permet de mettre en ordre les idées. Je n'écris pas pour parler avec quelqu'un mais j'écris pour moi-même »; ou encore "Quand on blogue, on découvre un nouveau sentiment : l'urgence d'écrire, notamment quand un sujet nous tient à cœur est mal traité ou peu traité ».

${ }^{66}$ Certains de ces blogueurs ont effectué une migration de retour au pays d'origine. C'est le cas, entre autres, des blogueurs Larbi et Hisham Al Miraat. Ces derniers se sont engagés dès leur retour au Maroc au sein d'associations nationales. Hisham Al Miraat a fondé et présidé l'Association des droits numériques (ADN). Cette association a été interdite par l'État marocain et ce blogueur de commencer un nouveau projet migratoire après ses déboires avec la justice marocaine.
} 
symbolique et l'absence de son efficacité auprès des politiques:

\begin{abstract}
"Y a-t-il équivalence entre "prendre la parole" et "prendre les affaires en main" ? [...] La [en référence à la prise de parole] croire efficace par elle-même, ce serait la prendre pour une chose et, par une sorte de magie, prétendre enchaîner les forces avec des mots, substituer des palabres au travail. Conclure de là qu'elle est insignifiante, ce serait perdre sens, remplacer par un mécanisme un système de relations et supposer finalement qu'une société peut fonctionner sans l'homme » (p. 38).
\end{abstract}

Les pratiques politiques en ligne, construites et circulant dans l'ensemble des dispositifs communicationnels étudiés, correspondent essentiellement à la production d'un discours critique et dénonciateur : elles prennent "la forme d'un refus » (De Certeau, 1994, p. 41). Elles se caractérisent par leurs créativités qui se donnent à voir à travers la plasticité de leurs formes d'écriture et à travers l'usage d'une diversité de procédés argumentaires.

\title{
Références bibliographiques
}

AIM O. et Y. JEANNERET, 2007, « L'encyclopédie de la parole possible: édition et scénographie politique sur I'Internet », Hermès, n 47, pp. 69-75.

AZIZI A., 2016, "Presse des immigrés marocains: entre construction identitaire et mobilisation politique ", $C^{\prime}$ mmunicati $n$ \& Langages, $n^{\circ} 187$, pp. 121-146.

BELGUENDOUZ A., 2006, Le traitement instituti nnel de la relati' $n$ entre les Mar' cains résidant à l'étranger et le Mar' $c$, Rapports de recherche, Institut universitaire européen, RSCAS, <http://cadmus.eui.eu/bitstream/handle/1814/6265/CARI M-RR2006_06.pdf>, dernière consultation le 19 août 2014.

BENCHENNA A., 2011, Fragiliser la contestation : les fidèles du Makhzen à l'assaut des réseaux », Médiam 'rph`se, $\mathrm{n}^{\circ}$ 30, pp. 87-90.

BENCHENNA A., 2014, "Médias et Marocains à l'étranger. Une préoccupation du pouvoir politique? », dans $\mathrm{T}$. 
Quand des immigrés prennent la par'le. Appropriation des plateformes de $b /$ ' gging à des fins politiques par des immigrés marocains

Mattelart (dir.), Médias et migrati' ns dans l'espace eur' méditerranéen, Paris, Mare \& Martin, pp. 149-182.

BERNAL V., 2006, "Diaspora, cyberspace and political imagination: The Eritrean diaspora », Gl` bal Netw`rks, vol. $6, n^{\circ} 2$, pp. 161-179.

BOURDIEU P., 1997, Ce que parler veut dire. L'éc' $n$ ' mie des échanges linguistiques, Paris, Fayard.

CALVET L.-J., 1993, La s`ci linguistique, Paris, Presses universitaires de France.

CAPPELLA J., V. PRICE et L. NIR, 2002, "Argument repertoire as a reliable and valid measure of opinion quality: Electronic dialogue during campaign $2000 »$, $P^{\prime}$ litical c' mmunicati ' $n$, vol. 19, $n^{\circ} 1$, pp. 73-93.

DAGHMI F., O. PULVAR et F. TOUMI, 2012, « Médias et publics au Maroc ", Les enjeux de l'inf' rmati' $n$ et de la c'mmunicati' $n, \mathrm{n}^{\circ} 13$, pp. 85-98.

DAGHMI F., 2014, "Diasporas dans les imaginaires et les pratiques des médias des pays d'origine ", dans C. AGBOBLI, O. KANE et G. HSAB (dir.), Identités diasp' riques et c'mmunicati' $n$, Québec, Presses de I'Université du Québec, pp. 91-105.

DAHLGREN P., 2012, "Web et participation politique : quelles promesses et quels pièges?", Questi ns de $c^{\prime}$ mmunicati $n, n^{\circ} 2$, pp. 13-24.

DANET B., 1998, "Flaming", dans P. BOUISSAC (dir.), Encycl' pedia 'f Semi' tics, New York/Oxford, Oxford University Press, pp. 246-247.

DAVALLON J., 1992, "Le musée est-il vraiment un média ? ", Publics et musées, $\mathrm{n}^{\circ}$ 2, pp. 99-123.

CERTEAU (de) M., 1994, La prise de par' le, Paris, Seuil.

DEL RIO L., 1996, Les micr' -médias imprimés : recherches sur la micr presse pendant la résistance chilienne 19731989 (thèse de doctorat), Université Paris La Sorbonne Nouvelle.

DESEILLIGNY O., 2008, « Du journal intime au blogue : quelles métamorphoses du texte?", C'mmunicati' $n$ \& Langages, $\mathrm{n}^{\circ} 155$, pp. 45-62.

DIMINESCU D., 2002, "L'usage du téléphone portable par les migrants en situation précaire ", $H^{\prime}$ mmes \& Migrati $n s$, n'1240, p. 66-79. 
DUPRET B., E. KLAUS et Z. GHAZZAL, 2010, « Commenter l'actualité sur Internet. La structure d'intelligibilité d'un forum de discussion arabe», Réseaux, n¹61, pp. 286317.

DUMONT A., 2008, «Representing voiceless migrants: Moroccan political transnationalism and Moroccan migrants' organizations in France », Ethnic and Racial Studies, vol. 31, n 4, pp. 792-811.

FLICHY P., 2001, L'imaginaire d'Internet, Paris, La Découverte.

JEANNERET Y., 2007, Y a-t-il (vraiment) des techn`I gies de l'inf 'rmati $n$ ?, Villeneuve d'Ascq, Presses universitaires du Septentrion.

HIDASS A., 2003, " La liberté d'opinion et d'expression au Maroc: normes, contingentements et transition démocratique », Annuaire de l'Afrique du N`rd, vol 39, Tome XXXIX, pp. 255-278.

KERBRAT-ORECCHIONI C., 1994, «Rhétorique et pragmatique : les figures revisitées ", Langue française, $\mathrm{n}^{\circ} 101$, pp. 57-71.

LACROIX T., 2005, " L'engagement citoyen des Marocains de l'étranger », $H^{`}$ mmes \& Migrati $n s, n^{\circ} 1256$, pp. 89-102.

LATZKO-TOTH G. et S. Proulx, 2013, « Enjeux éthiques de la recherche sur le web », dans C. BARATS (dir.), Manuel d'analyse du web en sciences humaines et $s$ 'ciales, Paris, Armand Collin, p. 32-48.

MARCOCCIA M., 2003, «Parler politique dans un forum de discussion », Langage \& s' ciété, $\mathrm{n}^{\circ} 104$, pp. 9-55.

MARION P., 1997, « Narratologie médiatique et médiagénie des récits ", Recherches en $c^{\prime}$ mmunicati $n, \mathrm{n}^{\circ} 7$, pp. 61-87.

MATTELART T., 2007, « Médias, migrations et théories de la transnationalisation », dans T. MATTELART (dir.), Médias, migrati ns et cultures transnati nales, Bruxelles, De boeck, pp. 14-56.

MATTELART T., 2009, « Les diasporas à l'heure des technologies de l'information et de la communication: petit état des savoirs ", tic\&s ciété, vol. 3, $\mathrm{n}^{\circ} 1-2$, $<$ https://ticetsociete.revues.org/600>, dernière consultation le 19 mars 2017.

MEMMI D., 1985, «L'engagement politique », dans M. GRAWITZ et J. LECA (dir.), Traité de science $p$ 'litique 
Quand des immigrés prennent la par'le. Appropriation des plateformes de $b l$ 'gging à des fins politiques par des immigrés marocains

( $v^{\prime}$ I. 3), Paris, Presses universitaires de France, pp. 310366.

MONNOYER-SMITH L., 2007, Les $v$ 'ies de l'expressi' $n$ cit' yenne dans les s'ciétés $m^{\prime}$ dernes. F'rmes et c'ntraintes des disp' sitifs du débat public (habilitation à diriger des recherches), Université Toulouse 1 Sciences sociales.

NAVARRO L., 2014, "Les médias nés des migrations marocaines en Espagne », dans T. Mattelart (dir.), Médias et migrati' ns dans l'espace eur'-méditerranéen, Paris, Mare \& Martin, pp. 319-362.

OUARDI S., 2006, "Publier la parole pour refonder le politique? Analyse d'un dispositif d'open-publishing ", $C^{\prime}$ mmunicati' $n$ et langages, $\mathrm{n}^{\circ} 147, \mathrm{pp} .61-71$.

PÈNE S., 2007, "Bloguer la politique », C’mmunicati” $n$ \& Langages, $\mathrm{n}^{\circ} 151, \mathrm{pp} .73-89$.

SCOTT J. C., 2008, La d'minati $n$ et les arts de résistance: fragments du disc'urs subalterne, Paris, Éditions Amsterdam.

SOUCHIER E., 1998, "L'image du texte : pour une théorie de l'énonciation éditoriale », Les Cahiers de médi' l' gie, $n^{\circ} 6$, pp. 137-145.

SOUCHIER E., Y. JEANNERET et J. LE MAREC (dir.), 2003, Lire, écrire, récrire. Objets, signes et pratiques des médias inf'rmatisés, Paris, Bibliothèque publique d'information.

TILLY C., 1984, "Les origines du répertoire de l'action collective contemporaine en France et en GrandeBretagne ", Vingtième siècle. Revue d'Hist'ire, $\mathrm{n}^{\circ} 4$, pp. 89-108.

TOUHAMI S., 2007, Pratiques médiatiques et pr`cessus d'intégrati $n$ : le cas de la $p$ ' pulati ' $n d^{\prime \prime}$ 'rigine mar' caine en France (thèse de doctorat), Université Michel de Montaigne-Bordeaux 3.

TRAUTMANN F., 2001, "Internet au service de la démocratie? Le cas d'ATTAC », Cahiers du CEVIPOF, $\mathrm{n}^{\circ} 30$, $<$ http://www.cevipof.com/fichier/p_publication/440/publica tion_pdf_cahierducevipof30.pdf>, dernière consultation le 19 mars 2017. 